\title{
Estimating the horizontal and vertical direction-of-arrival of water-borne seismic signals in the northern Philippine Sea ${ }^{\text {a) }}$
}

\author{
Simon E. Freeman ${ }^{\text {b) }}$ and Gerald L. D'Spain \\ Marine Physical Laboratory, Scripps Institution of Oceanography, 291 Rosecrans Street, \\ University of California San Diego, Scripps Institution of Oceanography, Marine Physical Laboratory \\ Building 4, San Diego, California 92106 \\ Stephen D. Lynch \\ SPAWAR Systems Center Pacific, Code 5528, 4297 Pacific Highway Building 7, San Diego, California 92110
}

\begin{abstract}
Ralph A. Stephen
Geology and Geophysics, Woods Hole Oceanographic Institution, 360 Woods Hole Road (MS24), Woods Hole, Massachusetts 02543
\end{abstract}

Kevin D. Heaney and James J. Murray

OASIS Incorporated, 5 Militia Drive, Lexington, Massachusetts 02421

Arthur B. Baggeroer

Massachusetts Institute of Technology, 77 Massachusetts Avenue, Room 5-206, Cambridge, Massachusetts 02139

Peter F. Worcester and Matthew A. Dzieciuch

Scripps Institution of Oceanography, Mail Code 0225, 9500 Gilman Drive, La Jolla, California 92093

\author{
James A. Mercer \\ Applied Physics Laboratory, University of Washington, 1013 Northeast 40th Street, Seattle, Washington 98105
}

(Received 3 December 2012; revised 1 May 2013; accepted 8 May 2013)

\begin{abstract}
Conventional and adaptive plane-wave beamforming with simultaneous recordings by large-aperture horizontal and vertical line arrays during the 2009 Philippine Sea Engineering Test (PhilSea09) reveal the rate of occurrence and the two-dimensional arrival structure of seismic phases that couple into the deep ocean. A ship-deployed, controlled acoustic source was used to evaluate performance of the horizontal array for a range of beamformer adaptiveness levels. Ninety T-phases from unique azimuths were recorded between Yeardays 107 to 119. T-phase azimuth and S-minus-P-phase timeof-arrival range estimates were validated using United States Geological Survey seismic monitoring network data. Analysis of phases from a seismic event that occurred on Yearday 112 near the east coast of Taiwan approximately $450 \mathrm{~km}$ from the arrays revealed a $22^{\circ}$ clockwise evolution of T-phase azimuth over $90 \mathrm{~s}$. Two hypotheses to explain such evolution-body wave excitation of multiple sources or in-water scattering - are presented based on T-phase origin sites at the intersection of azimuthal great circle paths and ridge/coastal bathymetry. Propagation timing between the source, scattering region, and array position suggests the mechanism behind the evolution involved scattering of the T-phase from the Ryukyu Ridge and a T-phase formation/scattering location estimation error of approximately $3.2 \mathrm{~km}$. (C) 2013 Acoustical Society of America.
\end{abstract}

[http://dx.doi.org/10.1121/1.4818843]

PACS number(s): 43.30.Ma, 43.60.Fg [JAC]

Pages: $3282-3298$

\section{INTRODUCTION}

The mechanisms by which seismic phases couple to the deep sound channel are highly dependent on bathymetry (Talandier and Okal, 1998; D'Spain et al., 2001; Park et al., 2001; Bohnenstiehl et al., 2003). Although studied for several decades, aspects of this coupling remain poorly

\footnotetext{
a) The results in this paper were presented at the Fall, 2011 Acoustical Society of America meeting.

b) Author to whom correspondence should be addressed. Electronic mail: sfreeman@ucsd.edu
}

understood (de Groot-Hedlin and Orcutt, 1999; Chapman and Marrett, 2006; Williams et al., 2006; Bohnenstiehl, 2007). T-phases ( $\mathrm{T}$ being an abbreviation for tertiary, following $\mathrm{P}$, primary, and $\mathrm{S}$, secondary, phases) are waterborne seismic waves created by $\mathrm{P}$ and $\mathrm{S}$ wave components propagating through and refracting, diffracting, and scattering from the ocean bottom into the water column. First described by Tolstoy et al. (1949), T-phases are frequently recorded by passive acoustic sensor systems in the deep ocean (e.g., Baggeroer et al., 2005). A definitive review of T-phase research is found in Okal (2008). Due to the highly efficient, continually refocusing nature of horizontal acoustic 
propagation in the deep sound channel, T-phase signals offer a method of seismic event detection and characterization for coastal and oceanic events that is far more sensitive than what can be achieved with a terrestrial seismometer network. Indeed, Dziak et al. (2004) have shown that the minimum Tphase detection threshold for deep sound-channel-based hydrophones is 1.53 lower in magnitude than that of terrestrial seismometer networks located around the Atlantic Ocean. Hence, T-phase analysis can yield unique insight into seismic events near and under the oceans. From an applied viewpoint, T-phase analysis is an important component of seismic monitoring systems such as for the Comprehensive Nuclear Test Ban Treaty (Harris et al., 1994; Okal, 2001).

Normal mode analysis of T-phase arrival structure has indicated that the first mode is often the dominant energy contributor (D'Spain et al., 2001). This mode travels with minimal bottom interaction and follows the most direct ocean-acoustic path from the T-phase formation site to the receiver. Unlike an ideal waveguide, in which the lowest order modes have the greatest group velocity, horizontal acoustic pathways in the deep ocean may not necessarily be the fastest due to higher sound speeds above and below the deep sound channel axis. Thus, precise knowledge of the water column sound speed profile along the travel path of the T-phase is very helpful when attempting to use the timedependent modal structure of T-phase arrivals to infer mechanisms of coupling to the deep sound channel (Jensen et al., 1994). The only significant T-phase attenuation mechanisms besides geometrical spreading are interaction with bathymetric features that protrude into the sound channel, and absorption by land masses. Such efficient propagation enables megameter-scale detection and characterization (Ewing et al., 1951; Johnson et al., 1963; Slack et al., 1999; Reymond et al., 2003; Okal, 2008). In addition, in shallow water such as continental shelf regions, earth-borne seismic energy is most likely to couple into the deep sound channel through the lowest order modes due to the depth-limited nature of higher order mode propagation (Jensen et al., 1994; D'Spain et al., 2001; Okal, 2008). It should be noted, however, that estimates of the epicentral location of seismic events that create $\mathrm{T}$-phases from $\mathrm{T}$-phases themselves are not definitive; significant variation can exist between epicenters and T-phase coupling site locations, the relationship between which is governed by many factors including bathymetry, crustal structure, and the type of seismic event. In this article, insight toward the complexities of such variation is provided through simultaneous horizontal and vertical measurement of T-phase arrival structure using hydroacoustic line arrays and adaptive beamforming algorithms. Evidence is presented to suggest that T-phase energy scatters from basin-scale bathymetric features, lengthening the duration of ensonification in the deep ocean.

The mechanisms of T-phase generation cannot be precisely known in each instance without accurate information regarding the originating seismic event, geoacoustic properties of the ocean bottom and sub-bottom, sea floor roughness, and bathymetric features within the coupling zone. Even if this information is known, it may not be available to the spatial resolution required for a complete assessment. Additionally, it is likely that not all mechanisms by which
T-phases couple to the deep sound channel have been discovered (Williams et al., 2006). Several mechanisms have been proposed to explain the formation of T-phases during transmission of seismic waves into the water column. Downslope conversion, described by Johnson et al. (1963), has been used to explain the coupling between body waves and the deep sound channel on sloping continental boundaries. Scattering from the rough sea floor in deep water has been proposed to explain the formation of T-phases far from any bathymetric features that substantially protrude into the deep sound channel (Walker et al., 1992; de Groot-Hedlin and Orcutt, 1999). Similarly, scattering from the rough sea surface may also enable entrainment of acoustic energy in the deep sound channel (Johnson and Norris, 1968; Keenan and Merriam, 1991). Additionally, direct refraction of seismic waves directly into the deep sound channel through vertical or near-vertical bathymetric features may result in the entrainment of acoustic energy at sufficiently small propagation angles to enable teleseismic (i.e., reception of a seismic signal more than $1000 \mathrm{~km}$ from the epicenter) transmission.

Evidence regarding the complex nature of T-phase generation mechanisms is indicated by the variation in the characteristics of T-phase arrivals on single-hydrophone or on terrestrial T-phase station recordings. Propagation effects in the deep ocean also influence these recordings. However, even from multiple locations, such recordings do not characterize the potential spatial variability of $\mathrm{T}$-phase coupling sites during an event. Additionally, they do not offer insight toward the vertical arrival structure of seismic phases.

The higher-frequency components of seismic events (greater than about $5 \mathrm{~Hz}$ ) typically are not recorded far from the hypocenter by terrestrial seismometers, as attenuation during propagation through the solid earth is strongly frequency dependent (Slack et al., 1999; Dziak et al., 2004). Provided that a given T-phase couples to the deep sound channel close to the hypocenter, the high-frequency components of the originating seismic event are more likely to be recorded by an in-water hydrophone as horizontal propagation of frequencies around 4 to $50 \mathrm{~Hz}$ is very efficient in the deep ocean. Consequently, more information regarding coastal and oceanic seismic events may be obtained through the use of water-column acoustic sensor systems than from recordings by terrestrial seismometers.

Analyses of T-phase arrivals using a hydrophone array capable of beamforming over the higher-frequency (approximately 5 to $50 \mathrm{~Hz}$ ) T-phase components confers unique advantages in T-phase characterization, detection and localization of the origin. Furthermore, knowledge to subwavelength scale accuracy of the relative positions of array elements and the array orientation allows for effective use of adaptive array processing. High-resolution data-adaptive techniques such as minimum variance distortionless response (MVDR) (Capon, 1969) and white noise constrained (WNC) beamforming (Cox et al., 1987) adaptively steer nulls toward off-look-direction, uncorrelated noise sources. The WNC technique can yield greater azimuthal resolution while retaining some robustness to violations of the assumptions made in array processing (Cox et al., 1987). Such techniques have been shown to improve the signal-to-noise ratio (SNR) of 
seismic phases (Harris et al., 1994). Combining simultaneous observations of T-phase arrivals from a horizontal array with another array oriented vertically reveals the azimuthal and modal nature of received T-phases (D'Spain et al., 2001). The ability to determine both horizontal and vertical characteristics of a water-borne seismic signal yields unique insight into the coupling of this energy through the oceanic crust into the deep ocean.

The objective of this article is two-fold; (1) to quantitatively evaluate the true azimuth-of-arrival estimation performance of the towed horizontal array deployed in deep water during PhilSea09 and (2) to examine the horizontal and azimuthal arrival structure of seismic phases recorded simultaneously on large-aperture horizontal and largeaperture vertical arrays in the deep ocean during PhilSea09. The next section of this paper briefly describes the experiment (Sec. II A) and acoustic sensor arrays used to collect the relevant data (Sec. II B). Section III outlines the data analysis approach including array processing methods (Sec. III B) and an automated T-phase peak-picking algorithm for rapidly obtaining angle-of-arrival estimates (Sec. III C). This section also describes the method by which the horizontal towed array true azimuth-of-arrival estimation performance with both conventional and data-adaptive beamforming algorithms was quantified using received tones from a controlled acoustic source at known azimuth, depth and range (Sec. III D). Results are presented in Sec. IV. The array azimuthal estimation performance results using the controlled source signals (Sec. IV A) are followed by a summary of the timeof-occurrence and azimuth-of-arrival of the 90T-phases identified in the PhilSea09 data set (Sec. IV B). Combined epicentral range and azimuth estimation is then evaluated using data from both the vertical and horizontal acoustic arrays for a seismic event characterized by the United States Geological Survey/National Earthquake Information Center (USGS/NEIC) seismic monitoring network (Sec. IV C). Evidence is then described of T-phase generation and/or scattering along the Taiwanese coast and Ryukyu Trench through the analysis of T-phase azimuthal and elevation angle of arrival characteristics recorded by both arrays (Sec. IV D). The observations indicate that the coupling of seismic energy into the deep sound channel can occur over large spatial scales well outside the margin of estimation error. Such observations have been made before (Chapman and Marrett, 2006) although not without refutation of the mechanisms involved (Bohnenstiehl, 2007; Chapman and Marrett, 2007). The results for this event's T-phase then are followed by a similar examination of the spatiotemporal arrival structure of its seismic body waves (Sec. IVE). Implications of observed seismic arrival characteristics are discussed in Sec. V. Finally, Sec. VI summarizes the conclusions from this work.

\section{DESCRIPTION OF THE EXPERIMENT AND SENSOR ARRAYS}

\section{A. Experiment}

A series of mesoscale acoustic experiments were performed in the Philippine Sea in April, 2009, as part of the
"PhilSea" experiment series (Worcester et al., 2010). In PhilSea09, the Five Octave Research Array (FORA) (Becker and Preston, 2003) was towed horizontally by the R/V Kilo Moana and the autonomous Distributed Vertical Line Array (DVLA) (Worcester et al., 2009) was bottom-moored at $21.365^{\circ} \mathrm{N}$ and $126.017^{\circ} \mathrm{E}$. Ship speed remained between 2.3 to $2.5 \mathrm{~ms}^{-1}$ for the duration of the experiment. Equipment was deployed from the R/V Melville, including the J15-3 controlled acoustic source. Figure 1 shows a map of the deployment locations. During periods of the experiment relevant to this study, the Melville was station-keeping $35 \mathrm{~km}$ from the location of the DVLA along a line bearing 17.5 degrees (position SS-35, 21.67 degrees N 126.12 degrees E). Figure 1 also illustrates the track taken by the R/V Kilo Moana towing the FORA on Yearday 112 from 04:20 to 10:15 UTC, on an arc centered at SS-35 and of radius $62.03 \mathrm{~km}$. This event is referred to as the "arc event." The DVLA and FORA recorded a number of seismic events during this time.

During the arc event, the controlled acoustic source was deployed at 15 and $60 \mathrm{~m}$ depths and produced a periodic signal of 5 min cycle duration. For $265 \mathrm{~s}$ of each cycle, the signal was comprised of seven tones, of which only the lowest

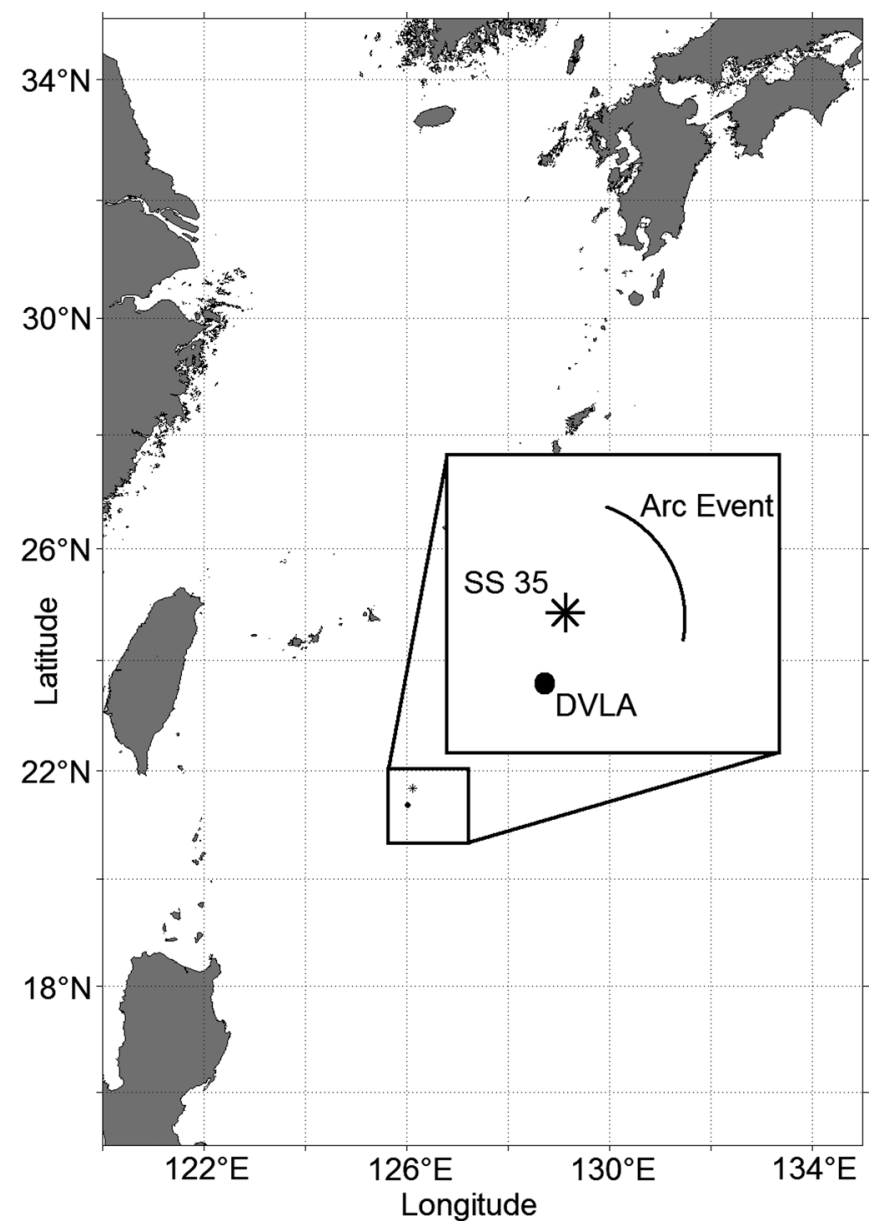

FIG. 1. This map of the PhilSea09 experimental site shows its proximity to Taiwan and the Ryukyu Island group. The filled circle represents the position of the DVLA, while the asterisk indicates the position of SS-35 where the R/V Melville deployed the J15-3 controlled acoustic source during the arc event (04:20-10:15 UTC, Yearday 112). The trajectory of the R/V Kilo Moana and the FORA during the arc event is represented by the arc to the east of SS-35. This arc was centered on SS-35 with a radius of $62.03 \mathrm{~km}$. 
three $(79,100,135 \mathrm{~Hz})$ were used in this study. Results from quantitative analysis of the azimuth of arrival of the lowestfrequency tones are presented in Sec. IV.

\section{B. Sensor arrays}

The FORA consists of five nested arrays of which four are linear, equally spaced hydrophone arrays designed with a range of inter-element spacings (Becker and Preston, 2003). The analysis of low frequency sounds presented in this paper was performed with data from a 16-element equally spaced sub-array consisting of every other element of the ultra low frequency (ULF) array, a component of the FORA with a design frequency of $250 \mathrm{~Hz}$. The inter-element spacing of this sub-array was $6 \mathrm{~m}$, corresponding to a design frequency of $125 \mathrm{~Hz}$. This sub-array is referred to as the ULF sub-array in this paper.

The array was towed $450 \mathrm{~m}$ behind the R/V Kilo Moana with an ULF array-center distance (element \#1) of $500 \mathrm{~m}$. FORA acoustic data were sampled at a rate of $8 \mathrm{Ksamples} / \mathrm{s}$ for all acoustic channels, with 24-bit analog-to-digital conversion. Conductivity, temperature, and depth (CTD) casts taken from the $\mathrm{R} / \mathrm{V}$ Kilo Moana indicated that sound speed at the average depth of the array was approximately $1520 \mathrm{~m} / \mathrm{s}$, corresponding to an in situ array design frequency of $126.7 \mathrm{~Hz}$. Non-acoustic sensor (NAS) packages were positioned on the FORA adjacent to elements \#1 and \#32 of the ULF array, a separation distance of $108.5 \mathrm{~m}$. Local magnetic bearing, roll, pitch, and yaw in addition to depth (resolved to $0.1 \mathrm{~m}$ ) were recorded at a sample rate of $3.906 \mathrm{~Hz}$ by the NAS. These data were used to calculate array pitch and array axis bearing, in addition to verifying array straightness as assumed in the beamforming algorithms. Periods during which array element position error due to curvature was greater than $10 \%$ of the wavelength of the maximum detected T-phase frequency (around $50 \mathrm{~Hz}$ ) were excluded from analysis. This $10 \%$ of wavelength error corresponds to a $1 \mathrm{~dB}$ reduction in array gain at that frequency using conventional beamforming (Hodgkiss, 1983). Shipboard GPS data from both the R/V Kilo Moana and the R/V Melville (with antenna positions near the center of the vessel) were recorded once a second throughout the experiment.

During PhilSea09 the DVLA (Worcester et al., 2009) was moored on the ocean bottom in approximately $5530 \mathrm{~m}$ of water. It consisted of two 1000-m-long, 30-element vertical sub-arrays, one spanning the sound channel axis located at about $1050 \mathrm{~m}$ depth, the other (the "deep sub-array") spanning the surface conjugate depth at around $4670 \mathrm{~m}$ for the month of April (Antonov et al., 2006; Locarnini et al., 2006). Note, however, that the conjugate depth varied significantly during PhilSea09 because of changing water properties within the surface mixed layer. The deep sub-array consisted of two nested, equal-element-spacing sub-arrays: a 20-element sub-array with $5 \mathrm{~m}$ inter-element spacing and a 12-element sub-array with $90 \mathrm{~m}$ spacing. The 12-element sub-array was centered on the estimated conjugate depth, while the 20-element sub-array (the "lower sub-array") was positioned entirely below the conjugate depth. DVLA acoustic data were sampled at 1953.125 samples/s at 24-bit resolution per channel. A high-pass resistor-capacitor (RC) filter with a pole at $10 \mathrm{~Hz}$ and a $6 \mathrm{~dB}$ /octave roll-off was incorporated as part of all DVLA acoustic channel data acquisition to remove very low frequency ambient noise and mechanical noise associated with cable vibration. The effects of this RC filter have not been taken into account in the results presented in this paper.

The DVLA was navigated using long-baseline navigation/interrogation/recording systems within the two array control modules positioned atop each 1000-m sub-array and four acoustic transponders deployed on the sea floor. Acoustic recordings were time-aligned with a dual-oscillator clock system on board each control module that was synchronized with GPS time. The maximum clock error during the experiment is approximately $1 \mathrm{~ms}$ (Worcester et al., 2009). DVLA data were recorded and stored independently on each hydrophone element. Time-alignment signals based on this clock system were transmitted inductively through the mooring cable to each element twice per hour to allow for post-experiment clock corrections. The resulting timing accuracy is more than sufficient for $\mathrm{P}, \mathrm{S}$, and T-phase range and azimuth estimation.

Time-of-arrival estimation was implemented using spectrograms from the deep sub-array. These data also were used to resolve the left-right ambiguity of the beamforming outputs from the FORA. Because of the relatively low-noise conditions around the conjugate depth, vertical beamforming was implemented using only the 20-element deep sub-array.

\section{DATA ANALYSIS}

\section{A. Spectral analysis}

Spectrograms for all 16 elements of the FORA ULF subarray were created using 50\% overlapped, 8192-point fastFourier transforms (FFTs) windowed with a Kaiser-Bessel function $(\alpha=2.5)$. For the DVLA, spectrograms were created for five elements, at 4285, 4915, 5205, 5245, and $5280 \mathrm{~m}$ depth. Due to the DVLA's lower data sampling rate, a 2048point FFT with $52 \%$ overlap was used to provide a nearly identical rate at which frequency domain "snapshots" were obtained. This approach allowed direct comparison between the DVLA and FORA arrays of the temporal variations in single-element spectra and beamformer output estimates.

All S-minus-P-phase and T-minus-P-phase range calculations were made from the DVLA mooring position. Comparisons between spectrograms of potential T-phase arrivals between DVLA elements, and between beamformer outputs from the FORA and DVLA arrays, were performed to rule out mechanical and electrical noise as spurious sources.

\section{B. Beamforming}

Narrow-band beamforming was implemented on data collected by the FORA ULF sub-array and the DVLA lower sub-array over all frequency bins from the first bin above $0 \mathrm{~Hz}$ to the spatial design frequency of each array (125 and $150 \mathrm{~Hz}$, respectively). Data cross-spectral density matrix (CSDM) estimates were averaged using $N+2$ snapshots, where $N$ represents the number of elements in the array, to 
ensure full rank of the CSDM before further processing. The resulting beamformer outputs were produced every $10 \mathrm{~s}$ for both the 16-element ULF sub-array and the 20-element DVLA sub-array.

Frequency-wavenumber analysis is a powerful method used to study the self-noise characteristics of towed arrays (Ferguson, 1998). Beamformer output magnitude squared, $B^{2}(\omega, k)$, on the frequency-wavenumber plane was calculated by multiplying plane-wave replica vectors and their Hermitian operators corresponding to spatial wavenumbers between $-k_{s} / 2$ to $k_{s} / 2\left(k_{s}\right.$ denotes the spatial sampling wavenumber) with the CSDM for each temporal frequency bin as shown in Eq. (1),

$$
B^{2}(\omega, k)=\boldsymbol{w}_{k}^{H}[\operatorname{CSDM}(\omega)] \boldsymbol{w}_{\boldsymbol{k}},
$$

where $\boldsymbol{w}_{\boldsymbol{k}}$ and $\boldsymbol{w}_{k}^{H}$ represent the plane wave vector and its Hermitian operator, respectively, for the $k$ th wavenumber. This process is equivalent to taking a spatial FFT of the complex Fourier coefficients in each temporal frequency bin. Broadband frequency-wavenumber outputs were examined to establish that non-acoustic noise levels were significantly lower than those within the frequency-wavenumber acoustic "cone" and that the cone position on the frequencywavenumber plane at wavenumbers $-k_{s} / 2$ and $k_{s} / 2$ corresponded to the sub array design frequency. This confirmed the array shape, element spacing, and provided an arrayderived estimate of in-water sound speed.

Frequency-azimuth beamformer outputs were used to determine the directionality and vertical characteristics of seismic phase arrivals at $10 \mathrm{~s}$ intervals. Frequency-azimuth plane-wave beamforming using conventional, MVDR (Capon, 1969), and WNC adaptive beamforming techniques (Cox et al., 1987) were used in analysis of acoustic data from both FORA and DVLA sub-arrays. To quantify the FORA azimuth-of-arrival estimation performance, an investigation was performed using the J15-3 acoustic signals that arrived near broadside during the arc event, when the exact location of the acoustic source was known. Estimates of the variance and bias of signal azimuthal estimates were produced for WNC beamformers representing a range of "adaptiveness," using constraint values of 0,6 , and $12 \mathrm{~dB}$ down from conventional. A constraint of $0 \mathrm{~dB}$ reduces adaptiveness in the WNC beamformer to zero, producing a result identical to one obtained using a conventional beamformer with a rectangular spatial window function, whereas $12 \mathrm{~dB}$ down creates a highly adaptive beamformer with characteristics approaching that of MVDR.

\section{T-phase directionality estimates}

Since T-phases propagating in the water column over long range typically have a predominantly first-mode structure (D'Spain et al., 2001), these arrivals are detected at horizontal incidence. Previous measurements of T-phases from vertical hydroacoustic arrays (Baggeroer et al., 2005) support this statement. The FORA pitch was no greater than $5^{\circ}$ during periods in which T-phases were recorded. The positions of individual array elements were estimated using the array pitch and depth measurements, and the assumption that the array was straight when the two compass headings recorded by the NAS differed by less than $2^{\circ}$. Array bearings relative to broadside were then converted to azimuthal estimates relative to true north, albeit with a left-right ambiguity along the axis of the array. Conversion from array-based bearings to true azimuth of arrival was implemented using 10 -s averages of the declination-corrected magnetic compass data from the two NAS packages. The left-right ambiguity was resolved by comparing the arrival times of individual seismic events between the FORA and DVLA. T-phases received during periods of time when one array was not recording were not considered in this analysis.

T-phases are characterized by azimuthally directional arrivals in the 2 to $50 \mathrm{~Hz}$ band, typically lasting between twenty and several hundred seconds. Although the T-phase pressure spectral density level decreases with increasing frequency, beamformer spatial resolution increases. As a result, typical T-phase arrival structures on the frequency/angle-ofarrival plane appear as a "triangle" of relatively high estimated pressure spectral density, as compared to background ocean noise. The wide "base" of the triangle is observed in the lowest frequency bins and covers the widest range of azimuths due to the comparatively poor azimuthal resolution of the array at these frequencies. Azimuthal resolution improves at higher frequencies, evolving to a point at high frequency (as in Fig. 9 presented later in the paper). The triangle is symmetric about an axis that represents the most likely azimuth of the T-phase. A peak-picking algorithm that exploits this triangular structure was employed to automatically and consistently estimate the directionality of T-phase arrivals over each 10-s beamformer output. Peak-picking was implemented over a range of estimated pressure spectral density magnitude contours from 34 to $52 \mathrm{~dB}$ re $1 \mu \mathrm{Pa}^{2} / \mathrm{Hz}$, typically spanning a frequency band from about 30 to $50 \mathrm{~Hz}$. Azimuthal estimates for each contour were averaged to obtain a mean T-phase arrival angle estimate relative to the FORA. T-phase arrivals recorded by the DVLA were evaluated in a similar manner to determine the elevation angle of arrival.

\section{Beamformer performance evaluation}

While the beamforming algorithms used to process the 79,100 , and $135 \mathrm{~Hz}$ tones produced by the J15-3 source for quantitative evaluation of beamforming performance are identical to those used in T-phase analysis, automated peakpicking of these tones in the frequency-azimuth plane required a different approach due to their narrowband nature. In addition, the J15-3 tones were obscured at times by transient noise most likely from local surface shipping. To minimize spurious estimates caused by this noise, the peakpicking results during times of low SNR were removed. Additionally, peak-picking was restricted to four frequency bins on either side of each expected J15-3 tone bin. Received levels of the source tones were significantly lower than the T-phase arrivals. Seven contour levels from 17 to $23 \mathrm{~dB}$ re $1 \mu \mathrm{Pa}^{2} / \mathrm{Hz}$, separated by $1 \mathrm{~dB}$, were used to obtain azimuthal estimates. Although the $135 \mathrm{~Hz}$ tone is above the design frequency of the ULF sub-array and spatial aliasing does occur, at look-directions close to array broadside the 
outputs are not affected by aliasing. To provide an indication of the variance of azimuthal estimates at lower frequencies, the in situ variances obtained for the 79, 100, and $135 \mathrm{~Hz}$ source tone azimuthal estimates were extrapolated down to those of T-phases using the frequency dependence derived in the following development. Assuming that the signal of interest is of sufficiently high SNR, the bearing resolution, $d \theta$, is such that the estimated bearing, $\hat{\theta}$, falls somewhere between the interval

$$
\hat{\theta}=\theta \pm \frac{1}{2} d \theta
$$

where the angle $\theta$ indicates the look angle from broadside to the axis of the line array $(\theta=0$ at broadside). Under the presumption that the probability of the true bearing occurring in this interval is given by the uniform probability density function (Pdf), then the expected bearing $E(\hat{\theta})=\theta$ and

$$
\operatorname{var}(\hat{\theta})=\frac{1}{12}(d \theta)^{2}
$$

The use of any other symmetric Pdf will only change the scaling factor of 1/12 in Eq. (3). Therefore, the variance of the bearing estimate is proportional to the square of the bearing resolution. The expression for the bearing resolution of a line array of length $L$ in the $z$ direction is

$$
d k_{z}=2 \pi / L
$$

where $k_{z}$ denotes wavenumber in the $z$ direction. Since $k_{z}=(\omega / c) \sin (\theta)=(2 \pi / \lambda) \sin (\theta)$, then

$$
d k_{z}=\frac{2 \pi}{\lambda} \cos (\theta) d \theta=\frac{2 \pi}{L} .
$$

Solving for $d \theta$ and incorporating Eq. (2) gives

$$
\operatorname{var}(\hat{\theta})=\frac{1}{12} \frac{\lambda^{2}}{L^{2} \cos ^{2}(\theta)} .
$$

This equation is similar to the expression for the Cramer-Rao bound (CRB) [Rao, 1945; Cramer, 1946, Eq. (1.25) in Tuncer and Friedlander, 2009], which is

$$
\mathrm{CRB} \approx \frac{1}{8 \pi^{2}} \frac{\lambda^{2}}{K(\mathrm{SNR}) \bar{d}^{2} \cos ^{2}(\theta)}
$$

However, as only an estimate of azimuth-of-arrival variance at lower frequencies was required, rather than an estimate of the minimum achievable variance, the $\lambda^{2}$ term in the numerator of both equations (6) and (7) is the critical information required for extrapolation. As such, both Eqs. (6) and (7) suggest that extrapolation of the standard deviation to lower frequencies relies on a $1 / f$ dependence. Such extrapolation was required as the only sound source available for array validation, the $\mathbf{J} 15-3$, is not capable of generating signals with sufficient energy below approximately $70 \mathrm{~Hz}$. Results of an analysis (not presented here) indicate that the relevant properties of the noise field between 79 and $135 \mathrm{~Hz}$ are approximately equivalent to those down to the lower frequencies of 20-30 Hz where peak-picking of the T-phase energy arrival angles is performed.

As illustrated by Eqs. (3) and (5), beamformer resolution, and in turn variance, are dependent on look direction with respect to broadside. Equation (5) shows how the resolution, $d \theta$, is proportional to $1 / \cos (\theta)$. Consequently, array variance and the $\mathrm{CRB}$ are proportional to $1 / \cos ^{2}(\theta)$. Note that for the beamformer performance evaluation, the controlled source tones all arrived at very near broadside so that $1 / \cos ^{2}(\theta) \approx 1$. However, as most T-phases arrived at FORA from larger offbroadside angles, the influence of beamformer resolution on bearing estimation must be taken into account.

To derive the azimuthal estimation error, the GPS-derived azimuth between the R/V Melville and the acoustic center of the FORA (ground truth) was subtracted from the azimuth of each tone estimated from the ULF sub-array data. Since the GPS information pertained to the position of the towing vessel rather than the array, compensation for the array displacement $500 \mathrm{~m}$ aft from the R/V Kilo Moana was first required. Figure 2 illustrates how the angular offset correction of $\theta=0.45^{\circ}$ was calculated. This correction was subtracted from estimated arrival angles during the arc event. A Lilliefors test for Gaussianity (Lilliefors, 1967) was performed on the resulting distributions of these errors, after removing those cases with low SNR, to verify that statistical inferences assuming a normal distribution could be made. Beamformer performance was not evaluated in the case of DVLA elevation estimates due to the lack of ground-truth information. Although the range, location, and depth of the J15-3 source were known, multipath propagation between source and receiver could not be numerically calculated with sufficient accuracy.

\section{RESULTS}

In the next subsection, results from the evaluation of FORA azimuth-of-arrival estimation performance, both with the controlled acoustic source data and a seismic event recorded on the USGS/NEIC seismic monitoring network, are presented. A general analysis of received T-phases is then given, followed by a detailed examination of a seismic event that was characterized by an unusual spatiotemporally evolving T-phase.

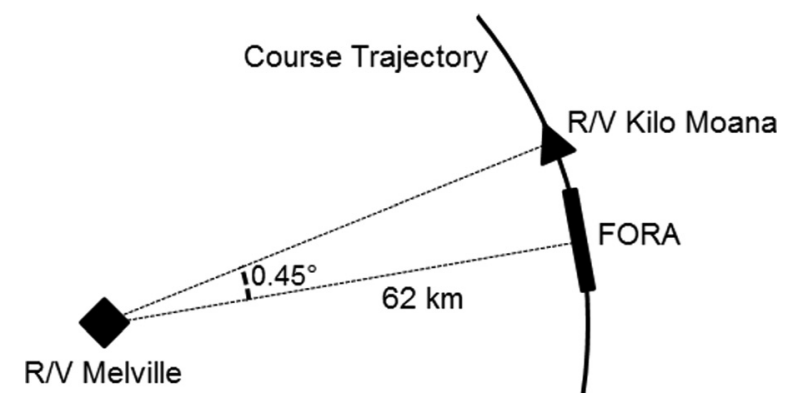

FIG. 2. This schematic shows the geometrical arrangement of the R/V Melville, R/V Kilo Moana, and the FORA during the arc event in PhilSea09. As the FORA position was inferred from the position of the R/V Kilo Moana, an angular compensation of $\theta=0.45^{\circ}$ was subtracted from the azimuthal estimates of the Melville-deployed controlled source in order to account for the $500 \mathrm{~m}$ distance between the R/V Kilo Moana and the FORA acoustic center. 


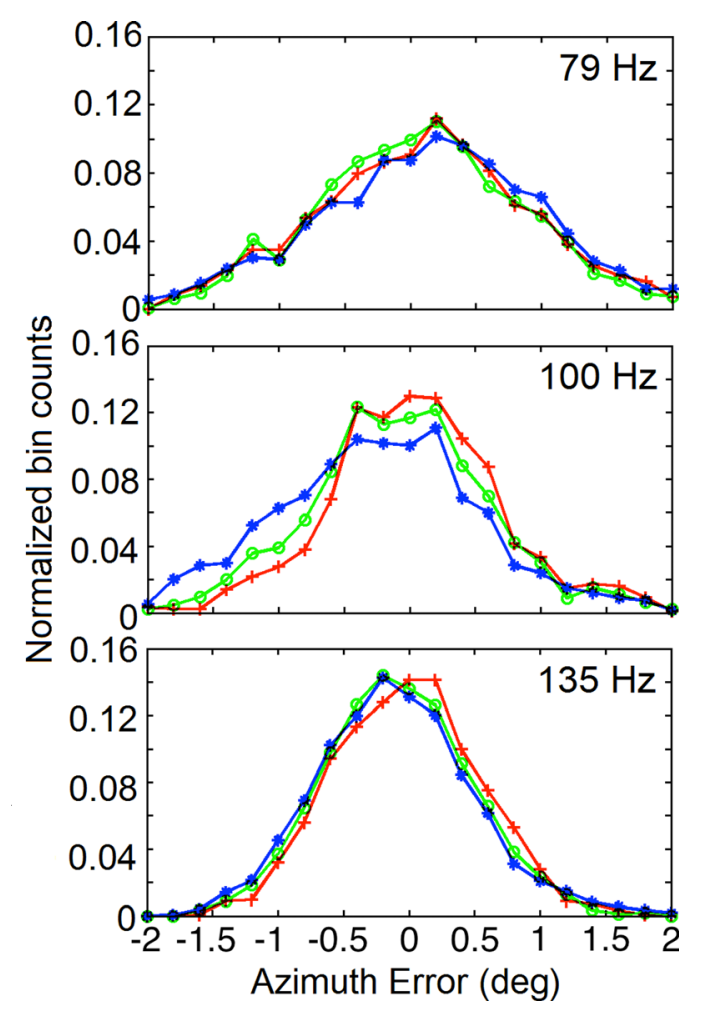

FIG. 3. (Color online) FORA direction-of-arrival estimation performance results for the conventional beamformer (stars), WNC $6 \mathrm{~dB}$ constraint (open circles), and WNC $12 \mathrm{~dB}$ constraint (plus signs) for the three J15-3 tones produced during the arc event are plotted in this figure. These azimuth error distributions were calculated by subtracting the GPS-derived azimuth from the beamformer-derived azimuth (corrected for magnetic declination and FORA offset as shown in Fig. 2). Bin quantities are normalized by the total sample size of each beamformer type/J15-3 frequency combination.

\section{A. Evaluating azimuth-of-arrival estimation performance using a controlled source}

Automated peak-picking of the arrival angles for the 79, 100 , and $135 \mathrm{~Hz} \mathrm{~J} 15-3$ source tones produced valid (i.e., within the specified frequency-azimuth window) estimates of azimuth of arrival for 90, 71, and $89 \%$ of the time during the arc event, respectively. Estimates could not be determined at other times due to the presence of interferers within the frequency bands of interest. Figure 3 shows the distribution of azimuthal estimation errors of each frequency for the three levels of beamformer adaptiveness. All beamformers produced mean source azimuth estimates within $1^{\circ}$ of the GPS-calculated azimuth over the 6 -h recording period. The arrival of the controlled source tone remained approximately

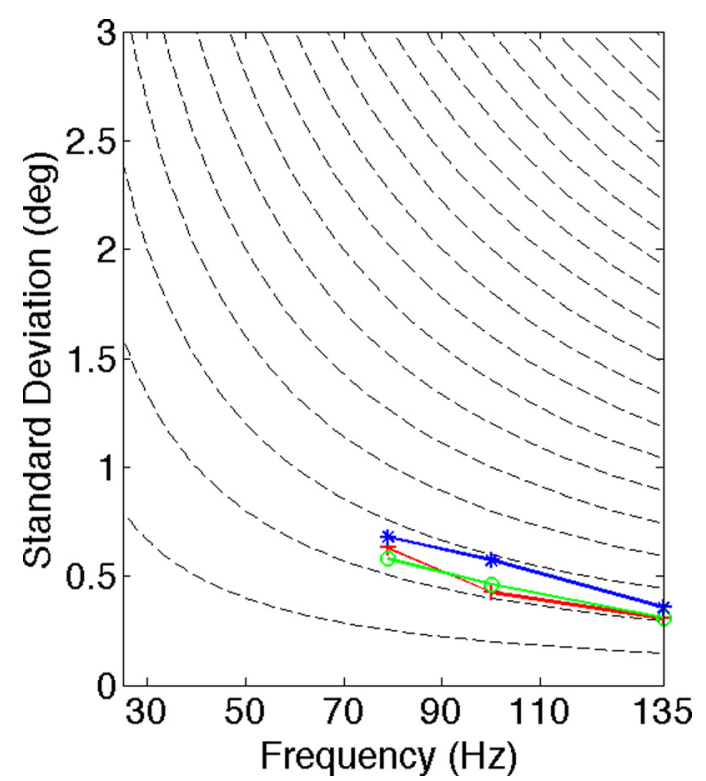

FIG. 4. (Color online) The standard deviation versus received frequency for the conventional (stars), WNC $6 \mathrm{~dB}$ constraint (circles), and WNC $12 \mathrm{~dB}$ constraint beamformers (plus signs) show an increase in standard deviation with a decrease in frequency. The dashed black lines show a succession of contours that follow a $1 / f$ dependence, used to conservatively estimate an approximate standard deviation at $30 \mathrm{~Hz}$ of at most $3^{\circ}$.

broadside to the FORA during this entire period, so that array resolution remained virtually unchanged during this evaluation. Table I displays the statistical values corresponding to the results shown in Fig. 3. The variance of the estimates for each beamformer increases with decreasing frequency in approximate agreement with the relationship in (6). Errors between the estimated azimuths of seismic events and USGS/NEIC epicentral data suggest Eq. (6) and the results shown in Fig. 4 provide a reasonable estimate of beamformer variance at the lower observed frequencies of T-phase energy for similar look directions.

Automated peak-picking of T-phase azimuths was only possible above $30 \mathrm{~Hz}$ due to the presence of a strong (35 to $40 \mathrm{~dB}$ re $1 \mu \mathrm{Pa}^{2} / \mathrm{Hz}$ ), spatially correlated, narrowband, persistent noise centered around $30 \mathrm{~Hz}$. As this noise always arrived from the end-fire forward direction, it most likely originated from the tow vessel R/V Kilo Moana. Consequently, a conservative extrapolated standard deviation at $30 \mathrm{~Hz}$, approximately $3^{\circ}$, was considered the standard deviation for T-phase azimuthal estimates. The variances of these three beamformer outputs show that adaptiveness has some improved ability to estimate source azimuth. Hence, results from the WNC

TABLE I. Number of estimates of azimuth of arrival (sample size), mean error (estimated azimuth minus GPS calculated azimuth between the FORA and J15-3 locations), and standard deviation from array, beamformer, and peak-picking algorithms during the arc event on Yearday 112, after removal of estimates with low SNR. "CBF" indicates the conventional beamformer result.

\begin{tabular}{|c|c|c|c|c|c|c|c|c|c|}
\hline \multirow[b]{3}{*}{ Received tone $(\mathrm{Hz})$} & \multicolumn{9}{|c|}{ Beamformer types (constraint values as dB level down from conventional) } \\
\hline & WNC 12 & WNC 6 & $\mathrm{CBF}$ & WNC 12 & WNC 6 & $\mathrm{CBF}$ & WNC 12 & WNC 6 & $\mathrm{CBF}$ \\
\hline & \multicolumn{3}{|c|}{ Number of detections } & \multicolumn{3}{|c|}{ Mean error (deg.) } & \multicolumn{3}{|c|}{ Standard deviation $(\sigma)$} \\
\hline 79 & 1181 & 1487 & 926 & 0.094 & 0.070 & 0.136 & 0.632 & 0.581 & 0.680 \\
\hline 100 & 871 & 1258 & 1338 & 0.041 & -0.072 & -0.233 & 0.426 & 0.464 & 0.574 \\
\hline 135 & 1253 & 1808 & 1839 & -0.019 & -0.087 & 0.104 & 0.305 & 0.306 & 0.356 \\
\hline
\end{tabular}


beamformer with a $6 \mathrm{~dB}$ constraint were used in the analysis of seismic signals. This "intermediate" level of adaptiveness provided a sufficiently narrow main lobe width to enable a reasonable degree of spatial resolution for the T-phase azimuth estimates, while producing a SNR that remained high enough for the peak-picking algorithm to be successful most of the time.

\section{B. T-phases during Yeardays 107-119, 2009}

Ninety T-phase arrivals over the period between Yeardays 107-119 were identified in FORA beamformer outputs by the peak-picking algorithm. The FORA left-right ambiguity of received $\mathrm{T}$-phases was resolved by comparing T-phase arrival times at the FORA and DVLA. Azimuthal estimates of these arrivals and the daily frequency with which they occurred during PhilSea09 are shown in Fig. 5. The left-right ambiguity could not be resolved for eight $\mathrm{T}$ phases that were recorded on Yeardays 110 and 116 when data from the DVLA were unavailable, and hence these Tphases are not included in Fig. 5. T-phase arrival azimuths were clustered around four regions: the southern terminus of the Ryukyu Trench and its intersection with the Gagua Ridge and Taiwan, the northern Ryukyu Trench to the west of the Okinawa Island group, the intersection between the Yap and Mariana Trenches, and the region of the Luzon Arc/Philippine fault. These regions correspond to areas in which a smaller number of earthquakes were detected by the USGS/NEIC seismic monitoring network during PhiSea09. These T-phases may thus have originated from smaller foreand after-shocks associated with larger events recorded by terrestrial seismometers.

\section{USGS/NEIC recorded event on Yearday 113, 2009}

Analysis of a seismic event that was of sufficient magnitude and proximity to land to be successfully recorded by the USGS/NEIC seismic monitoring network was performed in order to evaluate techniques used to determine range (Sminus-P-phase arrival time differences) and azimuth (FORA beamformer outputs). The event, of body wave magnitude (mb) 4.5, occurred on Yearday 113 with an estimated origin time of 16:09:56.66 UTC, at an epicenter located approximately $27.13^{\circ} \mathrm{N} 129.84^{\circ} \mathrm{E}$, and a hypocentral depth of $40 \mathrm{~km}$. This epicenter is $749 \mathrm{~km}$ from the DVLA, and approximately $200 \mathrm{~km}$ northeast of Okinawa Island along the Ryukyu Trench (Fig. 6).

S-minus-P and T-minus-P travel-time differences were calculated from the DVLA spectrograms and spectral ratios shown in Fig. 7. The International Association of Seismology and Physics 1991 (IASP91) earth model (Kennett and Engdahl, 1991) was then used to estimate range. An S-minus-P time difference of $72 \mathrm{~s}$ corresponds to an approximate range of $715 \mathrm{~km}$, using the USGS/NEIC hypocentral depth of $40 \mathrm{~km}$. The $34 \mathrm{~km}$ difference between the spectrogram-derived range estimate and the USGS/NEIC published range is approximately $5 \%$ of the epicentral range. This error likely arises from inaccuracies in applying the IASP91 model to this region of the globe, visually picking $S$ and P-phase arrival times, and errors in epicentral estimation
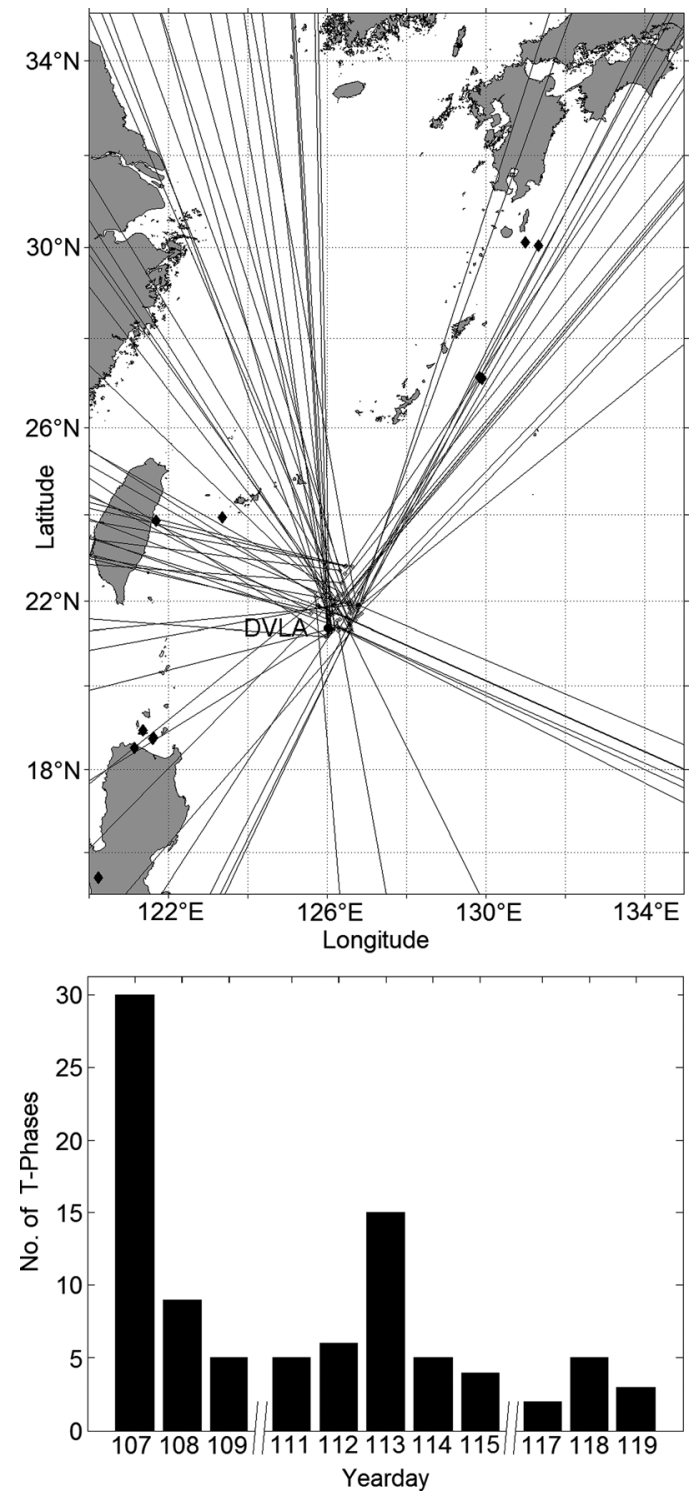

FIG. 5. The upper plot shows the azimuthal estimates for 82 of the $90 \mathrm{~T}$ phases recorded by the FORA between 00:00:00 UTC, Yearday 107 and 15:54:59 UTC, Yearday 119. Azimuthal estimates from Yeardays 110 and 116 are excluded due to unavailability of DVLA data. The solid circle indicates the mooring position of the DVLA. Solid diamonds indicate epicentral locations of eleven earthquakes estimated by the USGS/NEIC network during the PhilSea09 data recording period. The left-right ambiguity of the FORA was resolved through T-phase time-of-arrival differences between FORA and DVLA data. The histogram in the lower plot shows the number of T-phases received per day by the DVLA array during the experiment.

through the global seismometer network. Using the range based on the USGS/NEIC epicenter, the T-phase travel time of 8:25.34 s corresponds to a mean deep sound channel axis sound speed of $1482.4 \mathrm{~m} / \mathrm{s}$. This value is in good agreement with the mean axial sound speed of $1481.9 \mathrm{~m} / \mathrm{s}$ at $1100 \mathrm{~m}$ depth, determined through averaging 20 CTD casts taken during PhilSea09, in contrast to a value of less than $1415 \mathrm{~m} / \mathrm{s}$ obtained with the range calculated using DVLA S-minus-P travel-time differences.

Using the FORA ULF sub-array, the WNC beamformer with a $6 \mathrm{~dB}$ constraint, and the T-phase peak-picking algorithm, the highest received level of the $\mathrm{T}$-phase came in at an azimuth of $36.2^{\circ} \mathrm{T}$, indicating an offset of $4.4^{\circ}$ from the 


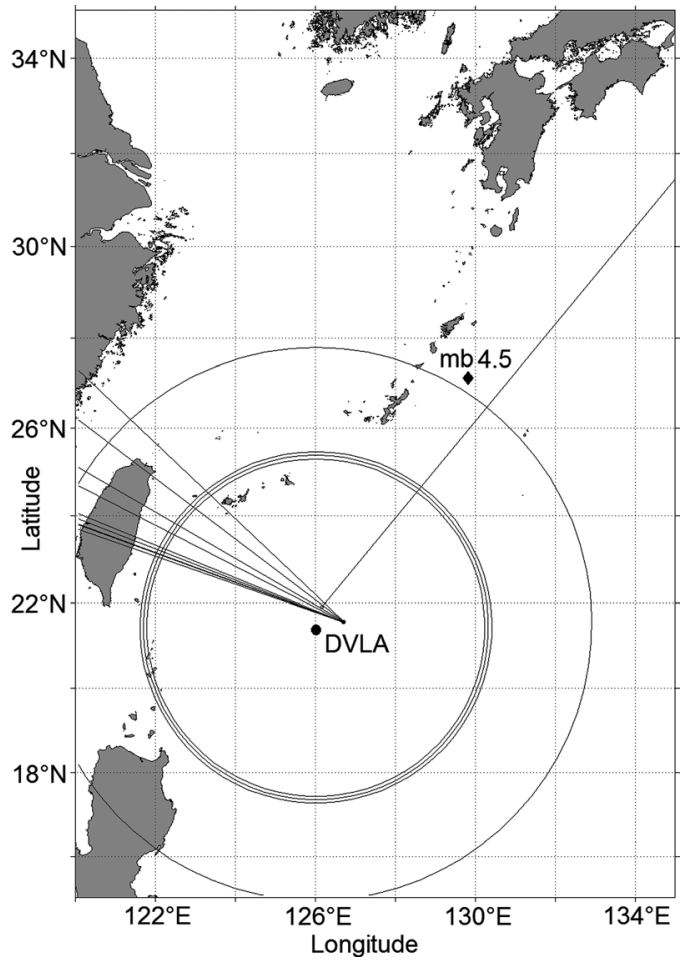

FIG. 6. Azimuthal estimates and DVLA-derived epicentral range estimates are illustrated for the mb 4.5 event that occurred at 16:09:56 UTC, Yearday 113 (for which the USGS/NEIC estimated epicenter is indicated by the solid diamond) and the event that occurred between 00:37:28 to 00:44:10 UTC on Yearday 112. The largest ring and the azimuthal line at $36.2^{\circ} \mathrm{T}$ represent the S-minus-P range and azimuthal estimates for the Yearday 113 event, respectively. The three smaller rings and the cluster of azimuthal estimates ranging from $291^{\circ}$ to $315^{\circ} \mathrm{T}$ represent the $\mathrm{S}$-minus-P range and azimuthal estimates for the Yearday 112 event, respectively. Each azimuthal estimate vector originates at the location of the FORA at the time each T-phase was received. The three range rings were calculated using assumed hypocentral depths from 10 to $40 \mathrm{~km}$, the largest ring corresponding to the deepest assumed depth. The nine azimuthal estimates indicate the angular variation observed during the single T-phase reception associated with this event. The DVLA position is indicated by the solid circle.

azimuth based on the USGS/NEIC published epicentral location, as shown in Fig. 6. The azimuth did not vary by more than $\pm 3^{\circ}$ from $36.2^{\circ}$ over the period during which the Tphase was discernable above ambient background noise. During reception of the T-phase signal, the FORA array was traveling on a bearing of $224.5^{\circ} \mathrm{T}$. As a result, the T-phase was received at a bearing of only $8.3^{\circ}$ from end-fire. Although the site of T-phase generation for this earthquake may be offset in azimuth from the epicentral location, this offset is too small to be resolved by the ULF sub-array given the increase in the variance of azimuthal estimates incident on the array at such angles [re Eq. (6)].

\section{Unique spatiotemporally evolving T-phase}

A seismic event recorded by both the FORA and DVLA on Yearday 112 between 00:37:28 and 00:44:10 UTC is the focus of the remainder of this section. DVLA single-element spectrograms spanning the conjugate depth and spectral ratios of this event are displayed in Fig. 8.

Comparisons between spectral ratios of the Yearday 113 event (Fig. 7, lower) and of this event show differing levels

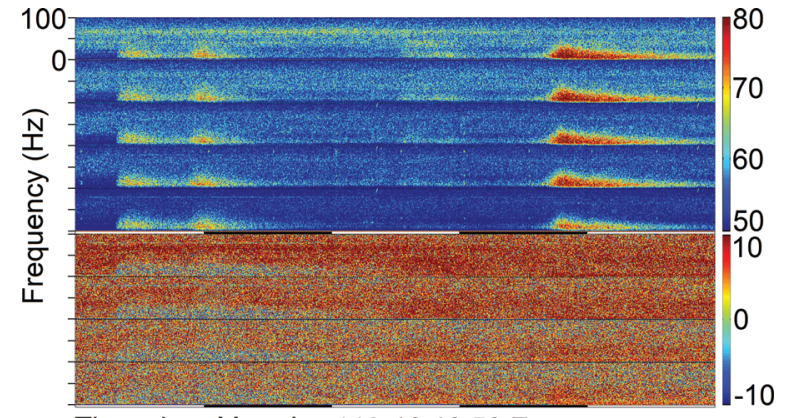

Time since Yearday 113 16:10:50 Z

FIG. 7. DVLA spectrograms from elements at 4285, 4555, 4825, 5005, and $5280 \mathrm{~m}$ (upper five plots) and spectral ratios between the upper four elements and the deepest element (lower four plots) show P-, S-, and T-phase arrivals associated with the seismic event that occurred at 16:09:56 UTC on Yearday 113. The frequency (vertical) axis of each spectrogram and spectral ratio is from 0 to $100 \mathrm{~Hz}$. Two-minute time periods are indicated by alternating black and white segments along the horizontal axes separating the spectrograms from the spectral ratios, and at the bottom of the spectral ratio plots. Color bars show pressure spectral density in $\mathrm{dB}$ re $1 \mu \mathrm{Pa}^{2} / \mathrm{Hz}$, (top right) and spectral ratio in $\mathrm{dB}$ (bottom right). $\mathrm{P}-, \mathrm{S}-$, and $\mathrm{T}$-phase arrival times are 16:11:28, 16:12:40, and 16:18:22 UTC, respectively.

of depth-dependence in the T-phase structures. While spectral ratios of the Yearday 113 event show some heterogeneity with depth, the ratio of pressure spectral densities is noticeably greater in the Yearday 112 event. The spectral ratios shown in Fig. 8 are further exaggerated by an increasing delay in onset time with depth that is less prevalent in Fig. 7.

The S-minus-P epicentral range estimates of 438,447 , or $456 \mathrm{~km}$ for this event were calculated based on assumed hypocentral depths of $10,20-30$, and $40 \mathrm{~km}$ in the IASP91 model, respectively. Circles corresponding to these three epicentral range estimates are shown in Fig. 6. An initial arrival azimuth of $292^{\circ} \mathrm{T}$ was measured by the FORA. This event was thus estimated to have occurred near the southwest terminus of the Ryukyu Trench, close to the east coast of Taiwan.

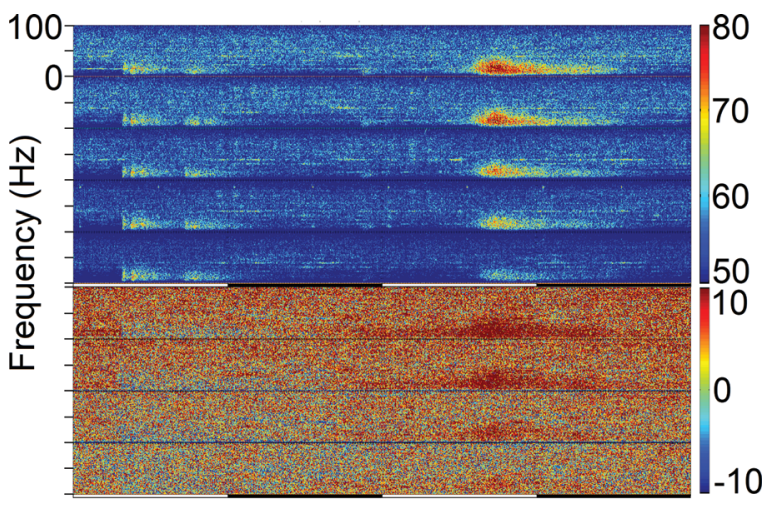

Time since Yearday 112 00:36:50 Z

FIG. 8. DVLA spectrograms and spectral ratios from the same elements as in Fig. 7 show P-, S-, and T-phase arrivals associated with the seismic event recorded between 00:37:28 to 00:44:10 UTC on Yearday 112. The frequency (vertical) axis of each spectrogram and spectral ratio is from 0 to $100 \mathrm{~Hz}$. Two-minute time periods are indicated by alternating black and white segments along the horizontal axes, as in Fig. 7. Color bars on the right of the plots have the same dynamic range and the same units as in Fig. 7. P, S, and T-phase arrival times are 00:37:28, 00:38:16, and 00:42:02 UTC, respectively. 
The DVLA spectrograms shown in Fig. 8 indicate a P-phase arrival at 00:37:28 UTC and an S-phase arrival at 00:38:16 UTC, with an S-minus-P time of $48 \mathrm{~s}$. Secondary $\mathrm{P}$ and S-phases were recorded between 5.5 and $7 \mathrm{~s}$ after the first arrivals on each element, with the time between the first and second arrivals in a pair being greater for the hydrophones closer to the sea floor. The corresponding T-phase arrival began at 00:42:02 UTC. A period of approximately $8 \mathrm{~s}$ elapsed between the T-phase onset at the hydrophones at 4285 and $5280 \mathrm{~m}$, and spectral ratios at these two depths indicate a greater than $10 \mathrm{~dB}$ decrease in T-phase energy below the conjugate depth compared to that above.

DVLA beamformer outputs reveal the temporal evolution of the vertical directionality of the T-phase arrival, shown in the right-hand column in Fig. 9. The first-arriving energy of the T-phase is in the horizontal plane. Although some noise centered at an elevation angle of $0^{\circ}$ exists before the T-phase, the estimated beamformed pressure spectral density level rises by $13 \mathrm{~dB}$ with T-phase onset. Levels are highest between 7 and $25 \mathrm{~Hz}$. The nature of the T-phase components in the 35 to $50 \mathrm{~Hz}$ band cannot be distinguished due to continuous, horizontally arriving ambient noise at this elevation angle. Approximately $40 \mathrm{~s}$ after the beginning of the T-phase, comparatively weak broadband signals arrive at elevation angles closer to end-fire. These arrivals are initially incident from $-50^{\circ}$ to $-90^{\circ}$ in elevation (coming from the sea floor) but subsequently begin to arrive from $+50^{\circ}$ to $+90^{\circ}$ (coming from the sea surface). Such off-horizontal arrivals continue to be received for as long as the T-phase is distinguishable from horizontally incident ocean noise, except for a 10 to $20 \mathrm{~s}$ hiatus that occurs around 00:43:20 UTC. Immediately after this hiatus, the off-horizontal arrivals gain in amplitude and become of equivalent level to the diminishing horizontal T-phase component. As the T-phase level diminishes, a $40 \mathrm{~Hz}$ tone of unknown origin appears as a horizontally incident signal.

FORA beamforming output, spanning the same time interval as the DVLA plots but starting $50 \mathrm{~s}$ later to account for the T-phase propagation delay between the FORA and DVLA arrays, is shown in the left column of Fig. 9. These plots show how the T-phase arrives from a relatively constant azimuth between 291 degrees and 293 degrees over the first $40 \mathrm{~s}$. T-phase spectral density levels were highest during this time, exceeding $50 \mathrm{~dB}$ re $1 \mu \mathrm{Pa}^{2} / \mathrm{Hz}$ over the 7 to $25 \mathrm{~Hz}$ band. Over the subsequent $50 \mathrm{~s}$, the estimated T-phase azimuth migrated clockwise from $294^{\circ}$ to $315^{\circ}$. Received levels remained clearly distinguishable from background noise during this time, although they decreased from approximately 45 to $30 \mathrm{~dB}$ re $1 \mu \mathrm{Pa}^{2} / \mathrm{Hz}$ in the $5-35 \mathrm{~Hz}$ band. As the Tphase was initially incident $12^{\circ}$ from broadside and ended $36^{\circ}$ from broadside, array resolution for each frequency bin decreased by $24 \%$. Higher frequency components (35-50 $\mathrm{Hz}$ ) peaked strongly in level during the initial $50 \mathrm{~s}$ but quickly diminished thereafter. About $90 \mathrm{~s}$ after its initial onset, the T-phase remains visible but is greatly reduced in amplitude, diffuse, and rendered indistinguishable to the peak-picking algorithm by low frequency noise emanating from the tow vessel.

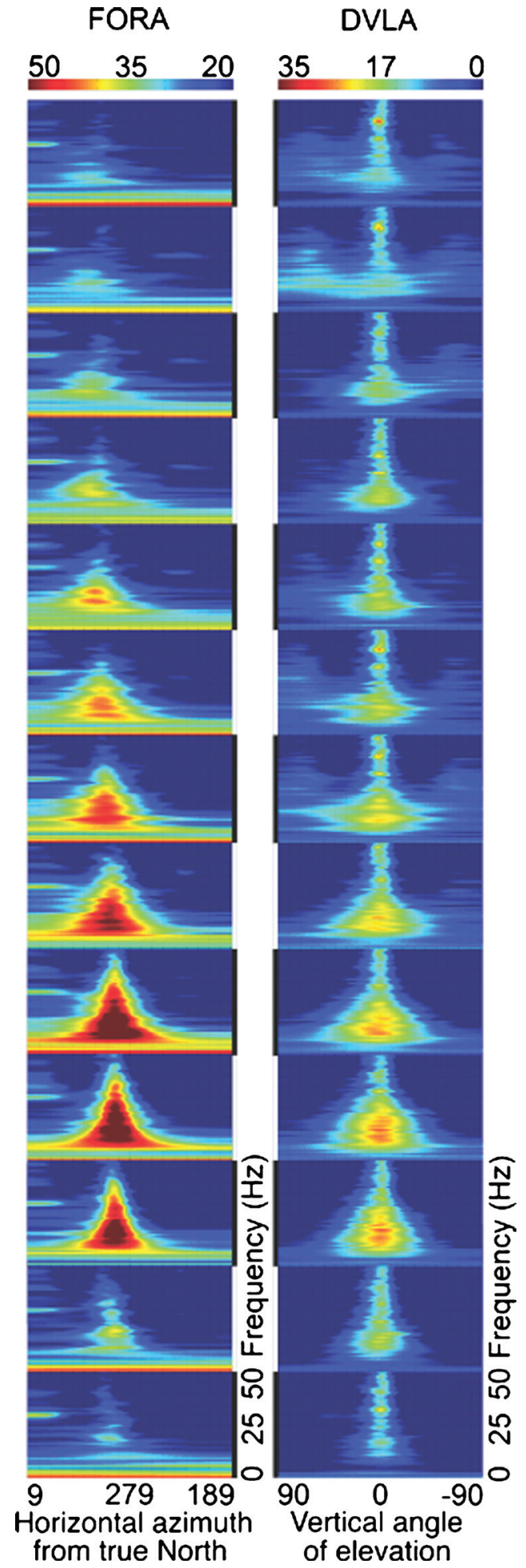

FIG. 9. A succession of frequency-azimuth plots from the FORA (left column) and DVLA (right column) shows the temporal evolution of the T-phase arrival associated with the event on Yearday 112. Beamformer outputs were created using the WNC beamformer with a $6 \mathrm{~dB}$ constraint level down from conventional. Each frequency/angle-of-arrival estimate represents a 10-s period beginning at 00:42:30 UTC for the FORA and 00:41:50 UTC for the DVLA. Thus, the two columns of rectangular plots each show $130 \mathrm{~s}$ of beamformer outputs as 13 subplots, each created from ten-seconds of data. The temporal order of the subplots begins at the bottom of the figure and proceeds upwards. The frequency axis for each ten-second plot is from 0 to $50 \mathrm{~Hz}$. Azimuth for the plots in the left column indicates angle (degrees) from true North. Elevation for the plots in the right column indicates vertical angle (degrees) from horizontal. Color bars show pressure spectral density in $\mathrm{dB}$ re $1 \mu \mathrm{Pa}^{2} / \mathrm{Hz}$. The color-scale is different for each array due to differing ambient noise levels. 


\section{E. Beamforming on $\mathbf{P}$ and $\mathbf{S}$ phases}

FORA and DVLA beamformer outputs for body waves associated with this seismic event reveal the two-dimensional spatial structure of these arrivals over time (Fig. 10). P- and $\mathrm{S}$-phases were observed as incident on the DVLA from a direction orthogonal to that from which T-phases from the same event arrived, consistent with previous $\mathrm{P}$ - and S-phase observations (Chapman and Marrett, 2006). These body phases are characterized as vertically propagating, broadband $(5-40 \mathrm{~Hz})$ arrivals that are approximately $30 \mathrm{~dB}$ greater in level than typical ocean noise in the vertical direction. The Pand S-phase arrivals persisted for approximately $10-40 \mathrm{~s}$ and showed a temporal evolution in the vertical plane, with arrivals initially incident at $-90^{\circ}$ (from the sea floor) followed by an arrival of similar frequency structure from end-fire at $+90^{\circ}$ less than $10 \mathrm{~s}$ later. Following the disappearance of the $\mathrm{P}$-phase, the S-phase is clearly discernible and also initially arrives from the sea floor. During P- and S-phase arrival, the characteristics of the horizontal noise field appear to remain largely unmodified, although the main lobes at frequencies of less than $30 \mathrm{~Hz}$ are sufficiently wide to obscure any horizontal arrivals during the occurrence of the main $\mathrm{P}$ - and $\mathrm{S}$-phase components. FORA beamformer outputs show these P- and S-phases arriving at broadside over the entire period they were discernible from ambient ocean noise. Although peaks in pressure spectral density of up to $38 \mathrm{~dB}$ re $1 \mu \mathrm{Pa}^{2} / \mathrm{Hz}$ were observed in the $5-20 \mathrm{~Hz}$ band, arrivals were spatially diffuse in elevation and quickly became indistinguishable from low frequency ambient noise.

\section{DISCUSSION}

\section{A. Overview}

The Philippine Sea region is an area that contains a high degree of tectonic activity due to the fact that the Philippine tectonic plate is surrounded on all sides by subduction zones, including the rapid subduction of the Philippine plate under the Eurasian plate, and the complex nature of associated plate interactions (Seno, 1977; Seno and Eguchi, 1983). A consequence of this activity is that the ocean acoustic field in the Philippine Sea frequently contains sounds from earthquakes located in multiple source regions, as shown in Fig. 5. Seismic signals that ensonify the entire water column often originate from local coupling of P- and S-phases at the sea floor, which then propagate predominantly in the vertical direction. Reflection at the surface and from the ocean bottom further extends the period of ensonification. T-phases travel in a predominantly horizontal direction parallel to the deep sound channel axis and arrive at a later time, but with greater pressure spectral density and over a typically wider frequency band (approximately $2-50 \mathrm{~Hz}$ wide in the highpass filtered FORA data acquisition system). T-phase arrivals may also be greatly extended in time as a consequence of the coupling mechanisms between the ocean bottom and the deep sound channel at the initial site(s) of T-phase generation, and to a lesser extent by the dispersive nature of sound propagation in the deep sound channel. Propagation within the deep sound channel is far more efficient than through the
FORA

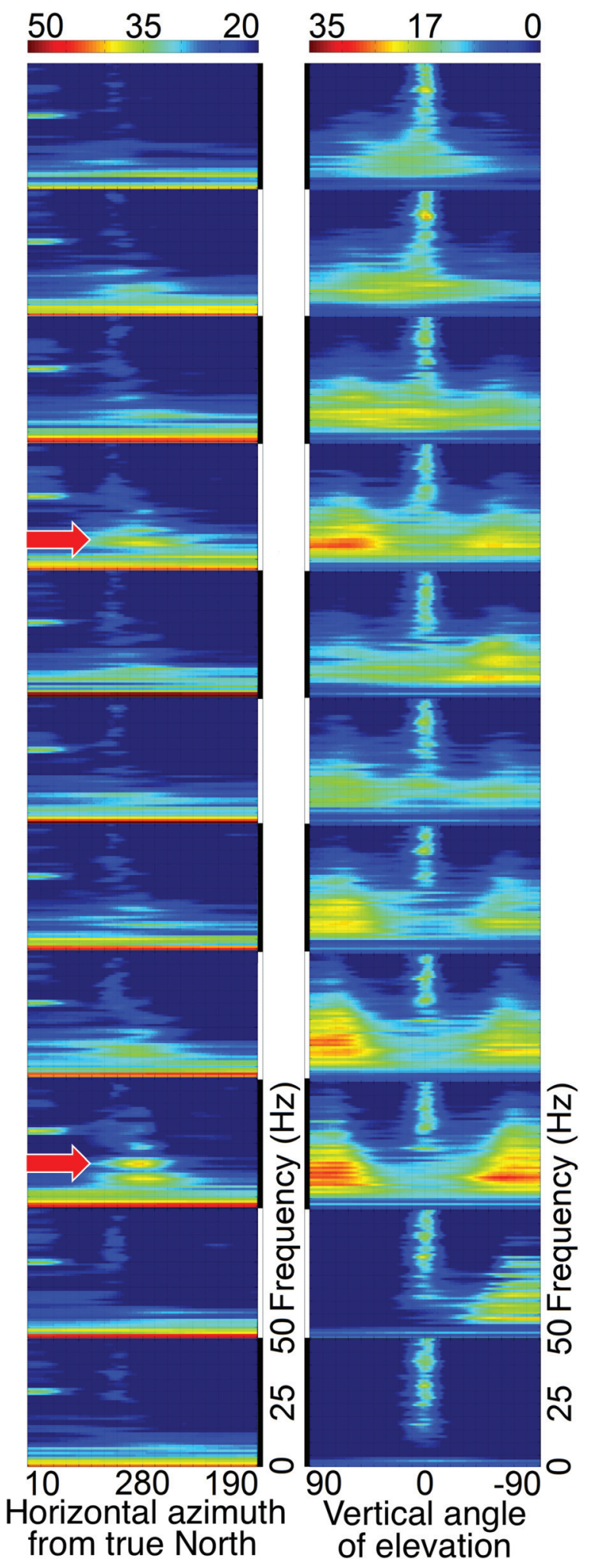

FIG. 10. A succession of frequency-azimuth plots from the FORA (left column) and DVLA (right column) shows the temporal evolution of P- and S-phase arrivals associated with the event that occurred on Yearday 112. The details of this plot are the same as in Fig. 9, except that the 11 subplots in each column represent $110 \mathrm{~s}$ of continuous data. Each frequency/angle-ofarrival estimate represents a 10-s period beginning at 00:37:40 UTC for the FORA and 00:37:10 UTC for the DVLA. The red arrows indicate interference bands created by the interaction between upward and downward traveling broad-band P- and S-phases. 
earth (Ewing et al., 1957; Brekhovskikh, 1960). This efficiency helps explain the occurrence of T-phases in the PhilSea09 array data sets likely associated with fore- and after-shocks that were too small to register on terrestrial seismometers. Although the process by which a T-phase is generated by seismic activity is highly dependent on the local bathymetry, characterization of the T-phase by hydroacoustic arrays may nevertheless reveal information regarding the source event that would not normally be available through $\mathrm{S}$ - and P-phase analysis using recordings from seismic sensors at regional and teleseismic ranges.

\section{B. Evaluation of direction-of-arrival estimation}

Array movement, oceanographic variability, beamformer resolution, and array processing limitations such as the minimum number of snapshots required to form each full-rank CSDM influence the mean azimuth-of-arrival error and the error variance with the FORA. Quantitative evaluation of the ULF sub-array using the J15-3 source tones determined the ability of this sub-array and associated signal/array processing algorithms to estimate the true azimuth-of-arrival of distant acoustic sources. Furthermore, analysis of three levels of beamformer adaptiveness elucidated the potential effect of off-look-direction interferers on azimuth error and variance. The fact that the tones from the J15-3 source were identified through the processing 90, 71, and $89 \%$ of the time (for the 79,100 , and $135 \mathrm{~Hz}$ tones, respectively) suggests that the FORA remained within the first convergence zone during the arc event in PhilSea09. Estimates of the single-tone azimuths showed that overall mean error near broadside was less than $1^{\circ}$ for all beamforming techniques and the variance generally decreased as adaptiveness and frequency increased. The effect of adaptiveness on SNR was not investigated.

The Lilliefors test for normality (at a 5\% significance level) indicated that the distribution of azimuth errors is likely Gaussian for all frequencies and beamformer adaptiveness levels except for the most adaptive (12 dB constraint) beamformer at $135 \mathrm{~Hz}$ (see Table II). A conservative extrapolation of the variance using the $1 / \mathrm{f}$ relationship to the lower frequencies of the T-phase arrivals indicated the standard deviation of single-frequency azimuthal estimates around $30 \mathrm{~Hz}$ to be approximately $3^{\circ}$. For the event discussed in Sec. IV D, the azimuths of arrival stayed within $2^{\circ}$ of the initial estimate of $293^{\circ}$ during the initial $50 \mathrm{~s}$, and then evolved over $22^{\circ}$ during the subsequent $40 \mathrm{~s}$, increasing in steps of $6^{\circ}, 9^{\circ}, 16^{\circ}$, and then $22^{\circ}$ from the original azimuth.

TABLE II. P-values from the Lilliefors test for Gaussianity performed on array, beamformer, and peak-picking algorithm outputs from data recorded during the arc event on Yearday 112, after removal of estimates with low SNR.

\begin{tabular}{lccc}
\hline \hline & \multicolumn{3}{c}{ Beamformer types } \\
\cline { 2 - 4 } Received tone $(\mathrm{Hz})$ & WNC 12 & WNC 6 & CBF \\
\hline 79 & $<0.001$ & $<0.001$ & $<0.001$ \\
100 & $<0.001$ & $<0.001$ & $<0.001$ \\
135 & Non-Gaussian & 0.4385 & 0.2935 \\
\hline \hline
\end{tabular}

Assuming the last four estimates actually came from a normally distributed population with a mean of $293^{\circ}$, the probabilities of obtaining these step increases in azimuth are $0.0228,1.35 \times 10^{-3}, 4.82 \times 10^{-8}$, and $1.12 \times 10^{-13}$, respectively. It is thus extremely likely that the T-phase azimuthal evolution over time represents a true physical phenomenon rather than being an artifact of array processing. Furthermore, estimation of T-phase azimuth involved the average of azimuth estimates across a broad band of frequencies (re the end of Sec. III C) as opposed to an estimate from a single-frequency tone. Consequently, the standard deviation of T-phase azimuthal estimates should be reduced from the pure-tone-derived value by dividing by a factor equal to the square root of the number of statistically independent frequency bins that comprise the average.

For the USGS/NEIC recorded seismic event on Yearday 113, the DVLA spectrogram-derived S-minus-P travel-time estimates, combined with the IASP91 earth model, produced epicentral range estimates that were 5\% smaller than the range calculated from the USGS/NEIC-estimated epicenter. The IASP91 earth model uses parameter estimates obtained from measurements under continental crusts, where the $\mathrm{P}$ and $\mathrm{S}$ wave speeds are typically lower than in oceanic crusts. Consequently, this model is likely to have contributed to the range estimation error. Nevertheless, this evaluation of Sminus-P epicentral range estimation using DVLA-derived spectrograms demonstrates that reasonable range estimates of seismic events not detected by the USGS/NEIC seismic network can be made under water. Combined with a T-phase initial azimuth-of-arrival estimate from the FORA array, an estimate of the event epicenter can be obtained. This additional step, however, assumes the initial part of the T-phase arrival coupled into the ocean directly above the hypocenter. The S-minus-P travel-time differences could also be measured in the FORA data, so that only a single array oriented in the horizontal is required. However, the increase in background noise at very low frequencies due to flow noise, towship noise, and noise trapped in the deep sound channel on a shallow, towed array greatly degrades the quality of the measurements compared to a stationary vertical array deployed below the conjugate depth.

\section{Spatiotemporally evolving T-phase event}

The epicenter of the event recorded between 00:37:28 to 00:44:10 UTC on Yearday 112 was estimated to be 438 to $456 \mathrm{~km}$ from the DVLA. Combined with the T-phase azimuthal estimates from the FORA, the estimated epicentral location is in the vicinity of the oceanic trough bordered by Taiwan to the west, the Gagua Ridge to the southeast and the Ryukyu Trench to the north. The apparent phase speed of the $\mathrm{P}$-wave at this epicentral range is much greater than the water column sound speed, indicating that the received Pphase coupled nearly vertically into the water column from the sea floor around the DVLA. Assuming a mean water sound speed of $1500 \mathrm{~m} / \mathrm{s}$, the surface reflection of the Pphase would take $7.04 \mathrm{~s}$ to return to the hydrophone positioned at $5280 \mathrm{~m}$ depth. The difference in arrival time between the direct arrival and its surface reflection decreases 
with decreasing hydrophone depth. Thus, the P- and S-phase "doublets" recorded by the DVLA (Sec. IV D) are a consequence of direct surface reflection. This conclusion is supported by DVLA beamformer outputs, which characterize these P- and S-phases as initially traveling upwards from the seafloor, followed later by downward propagation from the sea surface. In addition, an interference pattern created by the superposition of up and down-going phases near the sea surface is visible at broadside on the ULF sub-array beamformer outputs; Fig. 10 and third and eighth panels from the bottom in the left-hand column. For a FORA array depth of $125 \mathrm{~m}$, the predicted periodicity in frequency of this interference pattern is $6 \mathrm{~Hz}$, in good agreement with the spacing of the peaks in the third panel from the bottom of the left-hand column of Fig. 10. Spectral ratios of the DVLA elements at $4285,4915,5205$, and $5245 \mathrm{~m}$, normalized by the deepest element at $5280 \mathrm{~m}$, show an approximately $6-8 \mathrm{~dB}$ difference in received level between the deepest and shallowest elements, with the level being greatest at the deepest hydrophone. This result suggests some sort of geometrical spreading after local coupling between the sea floor and the water column. In comparison, T-phase received levels decrease with depth (see spectral ratios in Figs. 7 and 8). Consequently, while hydrophones below the conjugate depth may not record as much sound from some T-phases propagating in the deep sound channel as shallower hydrophones, $\mathrm{P}$ and S-phases are received at a greater pressure level below the conjugate depth.

Both the time of T-phase onset and T-phase spectral density levels also show a dependence on depth. Spectral ratios shown in Fig. 8 indicate an estimated pressure spectral density ratio greater than $10 \mathrm{~dB}$ in the 5 to $50 \mathrm{~Hz}$ band above the conjugate depth compared to that below for the T-phase associated with this event. In addition, approximately $8 \mathrm{~s}$ elapsed before the T-phase onset was detected at the deepest element of the DVLA compared to the shallowest element. Some energy may have arrived earlier at the deeper elements but remained undetected as it was below the level of ambient noise. This strong depth dependence indicates that the majority of the energy contained in this T-phase was restricted to the deep sound channel and therefore was comprised of the lowest order modes. In contrast, the T-phase arrival associated with the seismic event recorded on Yearday 113 did not show nearly as pronounced a variation in level with depth, as shown by the spectral ratio plots in Fig. 7. The published epicenter of this latter event is located in an area where the water depth exceeds $2500 \mathrm{~m}$, in contrast to the steep-sided bathymetry spanning the sound channel axis in the region where the Yearday 112 event is thought to have occurred. It is thus possible that the difference in depth dependent characteristics between the two T-phases is a consequence of bathymetry near the epicenter and hence the coupling mechanism that created the T-phase. Steep-sided bathymetry enables T-phase conversion to occur with fewer surfacebottom interactions (i.e., coupling into lower-order normal modes), and hence a reduced level of attenuation in propagating through the water column. Note, however, that because of the position of the Gagua Ridge, the highermode, deeper-reaching components of the Yearday $112 \mathrm{~T}$ - phase may have been physically obstructed by bathymetry that partially occluded the deep sound channel path between the source region and the DVLA. In any case, the relative received levels of seismic phases recorded along a largeaperture vertical array provide insight into the mechanisms that couple the propagating energy from the seafloor into the water column, and into the propagation conditions in the ocean. For the Yearday 112 event, the duration of the Tphase recorded by the hydrophone at $4285 \mathrm{~m}$ was approximately $110 \mathrm{~s}$, substantially longer than P- (at most $34 \mathrm{~s}$ ) and S-phase (at most $28 \mathrm{~s}$ ) arrival durations. As has been noted by many others, this increased duration is a testament to the complexity of the processes surrounding the coupling of seismic energy into, and efficient nature of subsequent propagation in, the deep sound channel.

The plots in Fig. 9 show the azimuthal progression and vertical structure of T-phase energy for the $90 \mathrm{~s}$ period during which an azimuth could be estimated from the beamformer output. Note that the T-phase is incident upon the array at an angle fairly close to broadside. Charting great circle paths from the FORA along these azimuthal estimates (Sec. IVD) suggests that the continental shelf east of Taiwan, the Ryukyu Trench, and the associated ridge/island group may be the sites of a progression of T-phase generation and/or scattering locations. Furthermore, S-minus-P time estimates do not place the epicenter further from the FORA than the coast of Taiwan or the Ryukyu Ridge. The depth dependent nature of the received T-phase levels suggest that the locations where they were generated were depth-centered around the deep sound channel axis at $1100 \mathrm{~m}$. Intersections of the great circle paths projected from FORA T-phase azimuth estimates with the $1100 \mathrm{~m}$ isobath are shown in Fig. 11. This figure shows the possible progression of source locations first northward, and then eastward with time.

T-minus-P time-based range estimates, using $\mathrm{T}$-phase arrival times taken at the point of maximum amplitude and a propagation speed of $1.48 \mathrm{~km} / \mathrm{s}$, combined with P-phase travel time calculations (again using the IASP91 model) suggest the range to the initial T-phase formation site to be approximately $518 \mathrm{~km}$. This range is significantly farther than the epicenter of the Yearday 112 event estimated using S-minus-P time calculations ( 438 to $456 \mathrm{~km}$ ). It is thus likely that the T-phase was initially formed closer to the east coast of Taiwan than the epicenter.

Over the first $40 \mathrm{~s}$ of the arrival, T-phase azimuth is approximately constant between $291^{\circ}$ and $293^{\circ}$. The steepsided continental shelf off the east coast of Taiwan is roughly perpendicular to the great circle paths of T-phase propagation between the hypothesized source regions and the location of the FORA and DVLA over this time. This bathymetry may have created T-phases from multiple locations close to the epicenter, leading to acoustic energy from a broad region of T-phase coupling locations arriving simultaneously at the FORA. The highest T-phase SNR was recorded over the first $40 \mathrm{~s}$ of the arrival. A high SNR also implies direct path propagation, a generation region(s) with closest proximity to the hypocenter, and higher efficiency of T-phase conversion. 


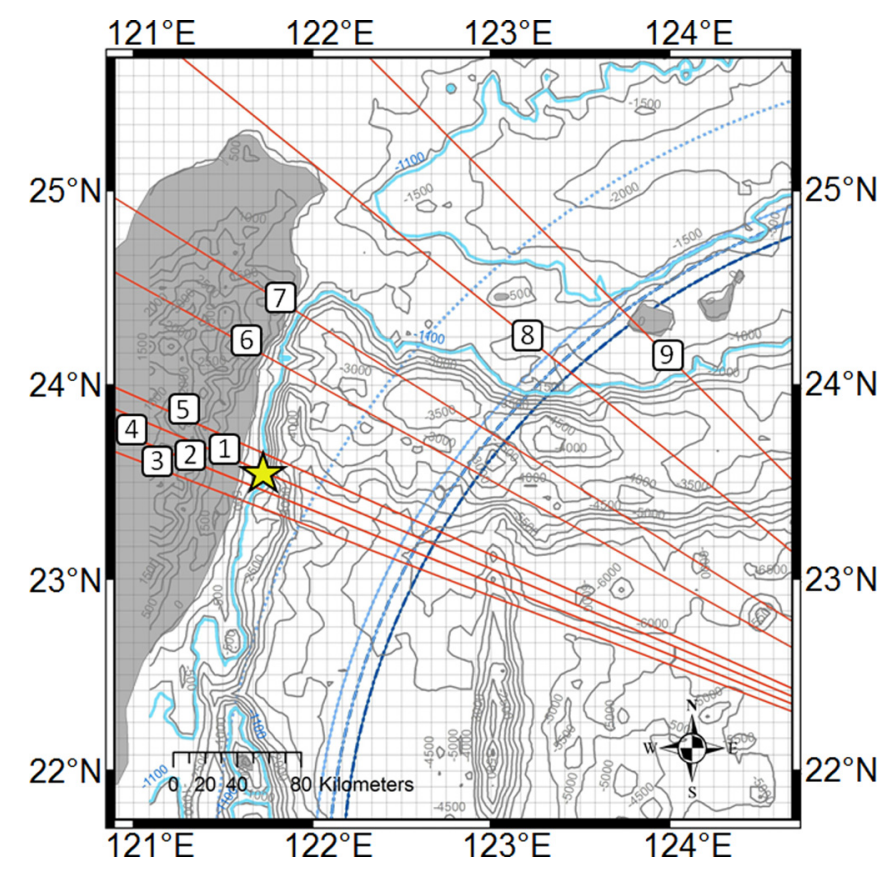

FIG. 11. (Color online) Beamformer-derived great circle paths (solid, straight lines) projected from azimuthal estimates of the T-phase received from 00:42:40 UTC on Yearday 112. Nine azimuthal estimates are shown numbered from 1 to 9 which correspond to $10 \mathrm{~s}$ increments from 00:42:40 to 00:44:00 UTC. The bathymetric contour emphasized in the map is the $1100 \mathrm{~m}$ isobath. The intersection of the $1100 \mathrm{~m}$ isobath and the beamformerderived great circle paths represent the estimated sites of T-phase formation or scattering, referred to in text by the azimuthal estimate number. Range circles show (from smallest to largest): Estimated range of seismic event epicenter based on $10 \mathrm{~km}$ depth, 20 to $30 \mathrm{~km}$ depth, $40 \mathrm{~km}$ depth, and approximate range of the T-phase coupling site based on T-minus-P time plus estimated P-phase propagation time calculated from the IASP91 solid earth model. The star represents a possible T-phase coupling location from which the strongest component of the received T-phase emanated. Azimuths \#2 and \#4 overlap at this scale.

The Gagua Ridge is a large underwater structure oriented north-south that rises from $5 \mathrm{~km}$ to less than $2 \mathrm{~km}$ depth along its length. It lies approximately $330 \mathrm{~km}$ west of the DVLA, about $140 \mathrm{~km}$ from the central east coast of Taiwan. The northernmost region in which the Gagua Ridge rises to within $2 \mathrm{~km}$ of the surface lies at $283^{\circ} \mathrm{T}$ from the FORA. North of this area, the ridge structure deepens to below $5 \mathrm{~km}$ depth before intersecting with the Ryukyu Ridge. Therefore, as the T-phase coupling sites moved northward towards the Ryukyu Ridge, the obscuring effect of the Gagua Ridge was reduced, facilitating the eastward propagation of a greater proportion of total $\mathrm{T}$ phase energy. This bathymetric effect may explain the delayed onset of T-phase energy at the greater depths of the DVLA. In contrast, T-phase components formed south of $291^{\circ} \mathrm{T}$ were likely to be at least partially blocked by the ridge.

In vertical elevation angle, the dominant component of the T-phase arrives broadside to the DVLA (Fig. 9, righthand column). The T-phase continues to arrive in the horizontal until approximately $40 \mathrm{~s}$ after the initial arrival when vertical components at a greatly reduced level begin to arrive. These off-broadside components persist as the dominant, horizontal component of the T-phase diminishes, until after $90 \mathrm{~s}$ from the onset, the horizontal and off-broadside components are approximately equal in level.
The off-horizontal arrivals beginning approximately 40 and $90 \mathrm{~s}$ after initial T-phase onset may indicate components of the T-phase that acquired a vertical component through scattering from the surface or smaller-scale deep bathymetric features. However, their similarity in frequency content, direction-of-arrival, and spatiotemporal characteristics to known P- and S-phase arrivals suggest that these arrivals could be due to a separate P- and S-phase train from an aftershock or an entirely independent event that arrived at the DVLA simultaneously with the T-phase. Whether these arrivals are associated with the T-phase or are P- and Sphases from an entirely different event cannot be resolved with the information at hand. However, no T-phases (from a hypothetical separate event) were recorded during the hour after the arrival shown in Figs. 8 and 9.

The precise mechanism that resulted in T-phase azimuthal migration cannot be determined conclusively from the available data since the range to each T-phase coupling site, and consequently an estimate of the actual locations of formation, cannot be determined. However, two hypotheses can be proposed to explain the azimuthally migrating nature of this T-phase.

\section{Hypothesis 1: Scattering}

The azimuthal evolution of the T-phase may result from in-water acoustic scattering from several locations of steepsided bathymetry along the northeast Taiwanese continental shelf and Ryukyu Trench/Ridge structure.

\section{Hypothesis 2: Body wave excitation}

The propagation of body waves from the hypocenter to the Taiwanese continental shelf and Ryukyu Trench/Ridge structure create, in progression, multiple T-phases from this one seismic event. (A similar train of T-phases could also be created by an event that propagates along a fault line, but the magnitude of such a seismic event corresponding to the spatial scale of T-phase azimuthal evolution would have to be unreasonably large.) This body wave excitation hypothesis requires the occurrence of a number of spatially independent coupling events to create the azimuthally evolving T-phase.

\section{Resolving the hypotheses}

To estimate the change in position of T-phase generation sites, the time required for each $\mathrm{T}$-phase component to reach the FORA from the estimated sites of T-phase formation along the $1100 \mathrm{~m}$ isobath, assuming propagation at $1481.9 \mathrm{~m} / \mathrm{s}$, was calculated. The speed with which the signal would need to propagate from either the site of initial $\mathrm{T}$-phase formation (hypothesis 1) or the region of the estimated hypocenter (hypothesis 2) to each hypothesized Tphase scattering/formation site on the $1100 \mathrm{~m}$ isobath was then calculated in order to satisfy the observed $10 \mathrm{~s}$ time difference between successive azimuthal estimates.

Hypothesis 2, that the creation of multiple, independent T-phases occurred from the same seismic event, is highly unlikely. As phases in the solid earth travel considerably faster than water-borne phases, a T-phase would have been 
produced at the intersection between the last azimuth $\left(315^{\circ}\right)$ and the $1100 \mathrm{~m}$ isobath — site \#9 in Fig. 11—approximately $15 \mathrm{~s}$ after the formation of the initial T-phase component off the east coast of Taiwan. Since site \#9 is $170 \mathrm{~km}$ closer to the FORA than the initial T-phase coupling site, the T-phase signal from the last azimuth estimate would arrive approximately $100 \mathrm{~s}$ before the first, rather than $90 \mathrm{~s}$ after. It is thus highly unlikely that the creation of independent T-phases at multiple sites from the same set of body waves explains the azimuthal evolution of the T-phase arrival in this case, although evidence exists to suggest that this phenomenon is possible given appropriate circumstances (Bohnenstiehl et al., 2003; Chapman and Marrett, 2006).

To test hypothesis 1 , an in-water propagation speed of $1481.9 \mathrm{~m} / \mathrm{s}$ was used. Although the T-phase azimuth remained within one standard deviation over the first $40 \mathrm{~s}$ of reception, the broadband $\mathrm{T}$-phase levels in each $10 \mathrm{~s}$ period first rose then fell, with the highest level occurring in the $30-40 \mathrm{~s}$ period. It is most likely that the strongest component of the initial $40 \mathrm{~s}$, rather than the first component, would be the predominant contributor to the later-arriving T-phase energy, particularly after interaction with the sea floor. A comparison of total travel times, assuming scattering at site \#9, was made for each of the first five azimuthal estimates. The results, shown in Table III, reveal that the in-water scattering hypothesis is most valid for scattering, from site \#9, of the T-phase components received by the FORA 40-60 s after the initial T-phase arrivals. The error for this period (the time difference between the arrival of these components and the estimated time based on the bathymetric scattering hypothesis) is less than the temporal resolution of the beamformer outputs (10s) and corresponds to a minimum source location estimation error of approximately $3.2 \mathrm{~km}$.

This analysis suggests that in-water scattering of $\mathrm{T}$ phase energy from steep-sided bathymetry can explain the observed T-phase azimuthal evolution. The change in amplitude and frequency content between the initial and scattered T-phases additionally support this hypothesis. That is, once

TABLE III. Time differences between T-phase component arrivals from the initial coupling location and the last estimated scattering location (\#9) for the seismic event on Yearday 112. The second column shows the time difference between FORA azimuthal estimates, while the third shows what the time difference would be under the assumption that the signal propagated from the site of initial formation to site \#9 and then to the FORA at $1481.9 \mathrm{~m} / \mathrm{s}$. The fourth and fifth columns show the time discrepancy and corresponding range error.

\begin{tabular}{lccrc}
\hline \hline $\begin{array}{l}\text { T-phase } \\
\text { azimuthal } \\
\text { estimate } \\
\text { number }\end{array}$ & $\begin{array}{c}\text { Measured time } \\
\text { difference (s) }\end{array}$ & $\begin{array}{c}\text { Time difference (s) } \\
\text { based on scattering at } \\
\text { site \#9 (see Fig. 11) }\end{array}$ & Error (s) & $\begin{array}{c}\text { Scattering } \\
\text { site location } \\
\text { error (km) }\end{array}$ \\
\hline 1 & 90 & 51.6 & 38.4 & 56.9 \\
2 & 80 & 44.5 & 24.4 & 36.2 \\
3 & 70 & 60.5 & 9.5 & 14.0 \\
4 & 60 & 55.6 & 4.4 & 6.6 \\
5 & 50 & 47.8 & 2.2 & 3.2 \\
6 & 40 & 29.1 & 10.9 & 16.1 \\
7 & 30 & 20.1 & 9.8 & 14.6 \\
8 & 20 & 13.5 & 6.5 & 9.6 \\
\hline \hline
\end{tabular}

propagating within the deep sound channel and unobstructed by bathymetric features, the high frequency components of a T-phase $(20-50 \mathrm{~Hz})$ are retained. In contrast, scattering from the sea floor results in significant attenuation of the higher Tphase frequency components (Stevens et al., 2001).

\section{CONCLUSIONS}

This study showcases the capabilities of simultaneously recording horizontal and vertical large-aperture hydrophone arrays along with adaptive array processing techniques to characterize higher-frequency $(4-50 \mathrm{~Hz})$ arrivals from seismic events. These data provide insight into T-phase formation and propagation mechanisms that cannot be determined using in-water single element or terrestrially located sensors.

Azimuth of arrival estimation performance near broadside was implemented using a 16-element sub-array (equal inter-element spacing for a design frequency of $125 \mathrm{~Hz}$ ) of the FORA towed horizontal array. Beamformers employing three different levels of data adaptiveness constraints for the 79,100 , and $135 \mathrm{~Hz}$ tones emitted by the J15-3 controlled source showed that the azimuth error distributions (1) passed the Lilliefors test for Gaussianity at the 5\% level of significance, (2) had approximately zero estimation bias, and (3) had standard deviations for the azimuth estimation error consistently less than $1^{\circ}$. The standard deviation was estimated to decrease with increasing frequency following an approximate $1 / f$ dependence. It also decreased slightly with increasing beamformer adaptiveness. A conservative extrapolated standard deviation of $3^{\circ}$ at $30 \mathrm{~Hz}$ for azimuth estimates of Tphase arrivals near broadside was estimated from the azimuth-of-arrival estimation variance at the higher controlled source tone frequencies, based on the proportionality between the variance of the bearing estimate and the square of the bearing resolution. During the 11 days of FORA array recordings in PhilSea09, 90 T-phases were identified and characterized. An automated peak-picking algorithm was developed to allow for rapid estimation of their true azimuth-of-arrival from the beamformer outputs. These 90 events clustered in space, arriving primarily from four different directions corresponding to the azimuths of events sufficiently large to be listed in the USGS/NEIC earthquake bulletin. Therefore, the majority of events identified in the PhilSea09 data set are likely fore- and after-shocks associated with these larger events. The USGS/NEIC-recorded event on Yearday 113 was used to evaluate the accuracy of hydrophone-array-derived epicentral range and azimuth. The DVLA spectrogram-based epicentral range estimate was 5\% smaller than that obtained using USGS/NEIC data, possibly because of a bias in the earth model (IASP91) used to convert measured P-minus-S travel-time differences into epicentral range. The T-phase travel time for this event corresponds to a mean deep sound channel axis sound speed of $1482.4 \mathrm{~m} / \mathrm{s}$, in good agreement with the CTD-derived sound speed minimum of $1481.9 \mathrm{~m} / \mathrm{s}$ at $1100 \mathrm{~m}$ depth. The azimuth of this event was estimated to be $36.2^{\circ} \mathrm{T}$ by the ULF sub-array, indicating an error of $4.4^{\circ}$ based on the USGS/NEIC published epicentral location. However, the Tphase was received at a relative bearing of only $8.3^{\circ}$ from 
end-fire, which substantially increased the expected variance of this azimuth-of-arrival estimate. Data from a seismic event recorded between 00:37:28 and 00:44:10 UTC on Yearday 112 suggests that T-phases can scatter and reverberate from prominent bathymetric features in the deep ocean, as observed by Northrop (1962). Such reverberation can cause the T-phase from a single seismic event to be received from multiple azimuths, increasing the duration of the Tphase arrival. A water-column hydrophone array with large horizontal aperture can help discriminate these scattered arrivals from those propagating along a direct path from the site of initial T-phase creation to the receiver, thus aiding in studies of T-phase generation.

Estimates of T-phase formation/scattering sites provide some insight into $\mathrm{T}$-phase generation and propagation in the Philippine Sea. However, the complex bathymetry that occurs in this region combined with the given physical array aperture and the variance of the array processing algorithms limited the ability to conclusively resolve the characteristics of these water-borne seismic phases. Mysteries remain about the precise mechanisms by which T-phases are created and various aspects of T-phase arrivals recorded by hydrophones remain unexplained (Williams et al., 2006). To further the analysis of T-phases using hydrophone arrays, a twodimensional horizontal array with a larger aperture for very low frequencies in both dimensions would enable triangulation of T-phase components, allowing for the location of multiple T-phase formation or scattering sites to be ascertained during the course of a continuous single T-phase arrival. Such information would enable more accurate and detailed event characterization, leading to more conclusive inferences regarding the mechanisms of T-phase formation, propagation, and scattering.

\section{ACKNOWLEDGMENTS}

The authors would like to thank Sean McPeak, formerly of the Marine Physical Laboratory, Scripps Institution of Oceanography (MPL/SIO) and now at the Applied Physics Laboratory, University of Washington (APL/UW), Keith von der Heydt (Woods Hole Oceanographic Institution-WHOI), and Kyle Becker at the Applied Research Laboratory, PA State University (ARL/PSU), for their leadership in the at-sea data collection effort. Eddie Scheer at WHOI made essential contributions both to at-sea data collection and postexperiment data analysis. The captains and crews of the $\mathrm{R} / \mathrm{V}$ Melville and R/V Kilo Moana were critical to the overall success of the experiment. Assistance in preparing the figures in this article was provided by Heidi Batchelor at MPL/SIO, Galina Rovner, also at MPL/SIO, helped with the initial processing of the DVLA data. Additional at-sea assistance was provided by Rex Andrew (APL/UW). This research is supported by the Office of Naval Research, both the Applied Research Laboratory program and Code 322(OA).

Antonov, J. I., Locarnini, R. A., Boyer, T. P., Mishonov, A. V., and Garcia, H. E. (2006). World Ocean Atlas 2005, Volume 2, edited by S. Salinity, NOAA Atlas NESDIS 62 (U.S. Government Printing Office, Washington, D.C.).
Baggeroer, A. B., Scheer, E. K., Colosi, J. A., Cornuelle, B. D., Dushaw, B. D., Dzieciuch, M. A., Howe, B. M., Mercer, J. A., Munk, W. H., Spindel, R. C., and Worcester, P. F. (2005). "Statistics and vertical directionality of low-frequency ambient noise at the North Pacific Acoustic Laboratory site," J. Acoust. Soc. Am. 117, 1643-1665.

Becker, K. M., and Preston, J. R. (2003). "The ONR five octave research array (FORA) at Penn State," Oceans 2003 MTS/IEEE, pp. 2607-2610.

Bohnenstiehl, D. R. (2007). "Comment on 'The directionality of acoustic Tphase signals from small magnitude submarine earthquakes,' J. Acoust. Soc. Am. 119, 3669-3675 (2006)," J. Acoust. Soc. Am. 121, 1293-1296.

Bohnenstiehl, D. R., Tolstoy, M., Smith, D. K., Fox, C. G., and Dziak, R. P. (2003). "Time-clustering behavior of spreading-center seismicity between 15 and 35 degrees $\mathrm{N}$ on the Mid-Atlantic Ridge: Observations from hydroacoustic monitoring," Phys. Earth Planet. Inter. 138, 147-161.

Brekhovskikh, L. M. (1960). Waves in Layered Media (Academic Press, New York).

Capon, J. (1969). "High-resolution frequency-wavenumber spectrum analysis," Proc. IEEE 57, 1408-1418.

Chapman, N. R., and Marrett, R. (2006). "The directionality of acoustic Tphase signals from small magnitude submarine earthquakes," J. Acoust. Soc. Am. 119, 3669-3675.

Chapman, N. R., and Marrett, R. (2007). "Reply to "Comment on 'The directionality of acoustic T-phase signals from small magnitude submarine earthquakes,' J. Acoust. Soc. Am. 119, 3669-3675 (2006)",' J. Acoust. Soc. Am. 121, 1297-1298.

Cox, H., Zeskind, R. M., and Owen, M. M. (1987). "Robust adaptive beamforming," IEEE Trans. Acoust., Speech, Signal Process. 35, 1365-1376.

Cramer, H. (1946). Mathematical Methods of Statistics (Princeton University Press, Princeton, NJ).

D’Spain, G., Berger, L., Kuperman, W., Stevens, J., and Baker, G. (2001). "Normal mode composition of earthquake T phases," Pure Appl. Geophys. 158, 475-512.

de Groot-Hedlin, C. D., and Orcutt, J. A. (1999). "Synthesis of earthquakegenerated T-waves," Geophys. Res. Lett. 26, 1227-1230, doi:10.1029/ 1999GL900205.

Dziak, R. P., Bohnenstiehl, D. R., Matsumoto, H., Fox, C. G., Smith, D. K., Tolstoy, M., Lau, T. K., Haxel, J. H., and Fowler, M. J. (2004). "P- and Twave detection thresholds, Pn velocity estimate, and detection of lower mantle and core P-waves on ocean sound-channel hydrophones at the Mid-Atlantic Ridge,” Bull. Seismol. Soc. Am. 94, 665-677.

Ewing, M., Press, F., and Worzel, J. L. (1951). "Further observations of the T-phase," Geol. Soc. Am. Bull. 62, 1527.

Ewing, W. M., Jardetzky, W. S., and Press, F. (1957). Elastic Waves in Layered Media (McGraw-Hill, New York).

Ferguson, B. G. (1998). "Minimum variance distortionless response beamforming of acoustic array data," J. Acoust. Soc. Am. 104, 947-954.

Harris, D. B., D'Spain, G. L., and Goldner, A. (1994). "Regional observation of a nuclear test from a vertical hydrophone array," Bull. Seismol. Soc. Am. 84, 1148-1153.

Hodgkiss, W. S. (1983). "The effects of array shape perturbation on beamforming and passive ranging," IEEE J. Ocean. Eng. 8, 120-130.

Jensen, F. B., Porter, M. B., Kuperman, W. A., and Schmidt, H. (1994). Computational Ocean Acoustics (American Institute of Physics, New York).

Johnson, R. H., Eppley, R., and Northrop, J. (1963). "Sources of Pacific T-phases," J. Geophys. Res. 68, 4251-4260, doi:10.1029/ JZ068i014p04251.

Johnson, R. H., and Norris, R. A. (1968). "T-phase radiators in the Western Aleutians," Bull. Seismol. Soc. Am. 58, 1-10.

Keenan, R. E., and Merriam, L. R. L. (1991). "Arctic abyssal T phases: Coupling seismic energy to the ocean sound channel via under-ice scattering," J. Acoust. Soc. Am. 89, 1128-1133.

Kennett, B. L. N., and Engdahl, E. R. (1991). "Travel times for global earthquake location and phase identification,” Geophys. J. Int. 105, 429-465.

Lilliefors, H. W. (1967). "On the Kolmogorov-Smirnov test for normality with mean and variance unknown,” J. Am. Stat. Assoc. 62(318), 399-402.

Locarnini, R. A., Mishonov, A. V., Antonov, J. I., Boyer, T. P., and Garcia, H. E. (2006). World Ocean Atlas 2005, Volume 1: Temperature (U.S. Government Printing Office, Washington, D.C.).

Northrop, J. (1962). "Evidence of dispersion in earthquake T-phases," J. Geophys. Res. 67, 2823-2830, doi:10.1029/JZ068i014p04251.

Okal, E. A. (2001). "T-phase stations for the international monitoring system of the comprehensive nuclear-test ban treaty: A global perspective," Seis. Res. Lett. 72, 186-196. 
Okal, E. A. (2008). "The generation of T waves by earthquakes," Adv. Geophys. 49, 1-65.

Park, M., Odom, R. I., and Soukup, D. J. (2001). "Modal scattering: a key to understanding oceanic T-waves," Geophys. Res. Lett. 28, 3401-3404, doi:10.1029/2001GL013472.

Rao, C. R. (1945). "Information and accuracy attainable in the estimation of statistical parameters," Bull. Calcutta Math. Soc. 37(3), 81-91.

Reymond, D., Hyvernaud, O., Talandier, J., and Okal, E. A. (2003). "TWave detection of two underwater explosions off Hawaii on 13 April 2000," Bull. Seismol. Soc. Am. 93, 804-816.

Seno, T. (1977). "Instantaneous rotation vector of Philippine Sea plate relative to Eurasian plate," Tectonophysics 42, 209-226.

Seno, T., and Eguchi, T. (1983), Seismotectonics of the Western Pacific region, in Geodynamics of the Western Pacific-Indonesian Region, Geodynamics Series, edited by T. W. C. Hilde and S. Uyeda (American Geophysical Union, Washington, D. C.), Vol. 11, pp. 5-40.

Slack, P. D., Fox, C. G., and Dziak, R. P. (1999). "P wave detection thresholds, Pn velocity estimates, and T wave location uncertainty from oceanic hydrophones," J. Geophys. Res., [Solid Earth] 104, 13061-13072, doi:10.1029/1999JB900112.

Stevens, J. L., Baker, G. E., Cook, R. W., D’Spain, G. L., Berger, L. P., and Day, S. M. (2001). "Empirical and numerical modeling of Tphase propagation from ocean to land," Pure Appl. Geophys. 158 $531-565$.
Talandier, J., and Okal, E. A. (1998). "On the mechanism of conversion of seismic waves to and from T waves in the vicinity of island shores," Bull. Seismol. Soc. Am. 88, 621-632.

Tolstoy, I., Ewing, M., and Press, F. (1949). "T-phase of shallow-focus submarine earthquakes," Geol. Soc. Am. Bull. 60, 1957.

Tuncer, E., and Friedlander, B. (2009). Classical and Modern Direction-ofArrival Estimation (Academic, Burlington, MA), pp. 35-39.

Walker, D. A., McCreery, C. S., and Hiyoshi, Y. (1992). "T-phase spectra, seismic moments and tsunamigenesis," Bull. Seismol. Soc. Am. 82, 1275-1305.

Williams, C. M., Stephen, R. A., and Smith, D. K. (2006). "Hydroacoustic events located at the intersection of the Atlantis $\left(30^{\circ} \mathrm{N}\right)$ and Kane $\left(23^{\circ} 40^{\prime} \mathrm{N}\right)$ transform faults with the Mid-Atlantic Ridge," Geochem. Geophys. Geosyst. 7, 1-28.

Worcester, P. F., Andrew, R. K., Baggeroer, A. B., Colosi, J. A., D'Spain, G. L., Dzieciuch, M. A., Heany, K. D., Howe, B. M., Kemp, J. N., and Mercer, J. A. (2010). "Acoustic propagation and ambient noise in the Philippine Sea: The 2009 and 2010-2011 Philippine Sea experiments," J. Acoust. Soc. Am. 128, 2385.

Worcester, P. F., Carey, S., Dzieciuch, M. A., Green, L. L., Horwitt, D., Lemire, J. C., and Norenberg, M. (2009). "Distributed Vertical Line Array (DVLA) acoustic receiver," in Proceedings of the 3rd International Conference on Underwater Acoustic Measurements: Technologies and Results, edited by J. S. Papadakis and L Bjørnø (Foundation for Research and Technology Hellas, Heraklion, Greece), 113-118. 